\title{
An efficient single-loop strategy for reliability-based multidisciplinary design optimization under non-probabilistic set theory
}

\author{
Xiaojun Wang ${ }^{\mathrm{a}, *}$, Ruixing Wang ${ }^{\mathrm{b}, \mathrm{c}, * *}$, Lei Wang ${ }^{\mathrm{a}}$, Xianjia Chen ${ }^{\mathrm{a}}$, Xinyu Geng ${ }^{\mathrm{a}}$ \\ a Institute of Solid Mechanics, Beihang University, Beijing 100191, China \\ ${ }^{\mathrm{b}}$ Key Laboratory for Mechanics in Fluid Solid Coupling Systems, Institute of Mechanics, Chinese Academy of Sciences, Beijing 100190, China \\ c School of Engineering Science, University of Chinese Academy of Science, Beijing 100049, China
}

\section{A R T I C L E I N F O}

\section{Article history:}

Received 22 February 2017

Received in revised form 15 November 2017

Accepted 28 November 2017

Available online 2 December 2017

\section{Keywords:}

Non-probabilistic reliability based

multidisciplinary design optimization

Single loop strategy

Uncertainty multidisciplinary analysis

Non-probabilistic reliability analysis

Translating distance calculation

\begin{abstract}
A B S T R A C T
Non-probabilistic reliability based multidisciplinary design optimization (NRBMDO) offers a powerful tool for making reliable decisions with the consideration of uncertain-but-bounded uncertainties for complex engineering systems. However, the prohibitive computation and convergence difficulties caused by the directly coupling of uncertainty based multidisciplinary analysis (UMDA), non-probabilistic reliability analysis (NRA) and MDO would seriously hamper the application of NRBMDO. In this paper, an efficient single loop strategy for NRBMDO (SLS_NRBMDO) is developed to decouple the nested issue and thus improve the computational efficiency. The key idea of the proposed strategy is decoupling NRBMDO with several cycles of sequential MDO, UMDA, NRA and translating distance calculation (TDC). For UMDA, three methods, i.e., the first order interval Taylor expansion method, the interval vertex theorem, the direct optimization approach are formulated. Besides, NRA is conducted on the basis of the expanded non-probabilistic stress-strength interference model and the volume ratio thought, which provides a clear and definite assessment criterion for the structural safety with uncertain-but-bounded parameters. Furthermore, the translating strategy based on the performance measure approach is proposed to shift and update the constraints, and the expression of the translating distance is mathematically derived to accelerate the design procedure. Eventually, the effectiveness and efficiency of the proposed method are illustrated with one numerical case and one practical supersonic wing optimization design problem.
\end{abstract}

(c) 2017 Elsevier Masson SAS. All rights reserved.

\section{Introduction}

Due to the increasing complexity of modern structural systems, multidisciplinary design optimization (MDO) is widely acknowledged as an effective approach to designing the coupled engineering systems in recent years. Numerous successful examples of MDO applications have been reported in complex engineering systems, such as aerospace system [1,2], vehicle design [3,4] and fluid-structure problem $[5,6]$. The input variables in the traditional MDO are assumed to be non-varying and the multidisciplinary system are assumed to behave as an analysis model predicts [7]. However, large scale structures are always significantly influenced by plenty of uncertain factors included in model, specification, environment and so on [8]. Thus, reliability is an important issue in the

\footnotetext{
* Corresponding author.

** Corresponding author at: Key Laboratory for Mechanics in Fluid Solid Coupling Systems, Institute of Mechanics, Chinese Academy of Sciences, Beijing 100190, China.

E-mail address: wangruixing@imech.ac.cn (R. Wang).
}

design phase that should be addressed by comprehensively taking potential uncertainties into account [9]. To ensure the high reliability in complex systems design, techniques of reliability based MDO (RBMDO) have been recently developed as advanced methodology to address the multidisciplinary system with uncertainties [10-12].

The conventional approach for solving a reliability-based optimization (RBO) problem is to conduct a double-loop strategy. The reliability analysis and the deterministic optimization are nested in such a way that the inner loop performs the reliability analysis and the outer loop executes the optimum search. Obviously, the double-loop strategy may lead to unbearable calculation amount in actual engineering structures. Therefore, various techniques have been developed to improve the efficiency. For example, the sensitivity analysis methods $[13,14]$ have been used to reduce the design variables and uncertain parameters to decrease the complexity. Approximation methods have been used to replace the complicated disciplinary analysis model with simple approximate functions to cut down the calculation. In addition, considering the computational burden attributed to the traditional double-loop procedure, several strategies [15-21] have been proposed to decouple the nested process into a single level procedure. Among 
these, the sequential optimization and reliability analysis (SORA) [20] method formulates the deterministic constraint by shifting the inverse most probabilistic point at the current design at least onto the deterministic boundary which ensures the constraint feasibility in the next deterministic optimization satisfying required reliability. The safety-factor based approach [21] converts the reliability constraints into the equivalent deterministic constraints with safety factors and the sequential optimization strategy is applied. The high computational efficiency of single loop strategies was testified by numerical examples compared with conventional double-loop procedures.

Based on the single loop strategy in RBDO, many researchers focus on applying this decoupling theory on the RBMDO problems to reduce the huge calculation caused by the coupled multidisciplinary system, and many significant achievements have been made. Du et al. [22] proposed a methodology of sequential optimization and reliability assessment for MDO to improve the efficiency of RBMDO, in which the reliability analysis is based on the first-order reliability method (FORM). Li et al. [23] proposed a sequential multidisciplinary reliability analysis (SMRA) approach that integrates the concurrent subspace optimization strategy and the performance measure approach, in which the multidisciplinary analysis, system sensitivity analysis and reliability analysis are decoupled and arranged sequentially. Zhang and Huang [24] proposed formulations of mixed variables (random and fuzzy variables) multidisciplinary design optimization, and then a method within the framework of sequential optimization and reliability assessment was also presented.

Note that, most studies of RBMDO are based on the probability theory which needs sufficient information to determine the probability density function. Nevertheless, experimental samples are often inadequate in practical engineering. Compared with the probability theory, the non-probabilistic theory only needs the approximations of the bounds of uncertain information, which is well suitable for the poor information situations. Considering that, the non-probabilistic theory was proposed to deal with the analysis and optimization problems with limited uncertain information and the original investigations were conducted by Ben-Haim and Elishakoff [25-27] in the early 1990s. Since then, the nonprobabilistic theory has been further developed and widely applied in both practical engineering and theoretical research [28-32].

As for the non-probabilistic reliability issue, the reliability indexes can be divided into two sorts. For one thing, the minimum distance quantified by the infinity norm from the origin to the failure surface is considered as a measure of non-probabilistic reliability by Kang et al. [33] and Guo et al. [34]. This reliability index is similar to the first-order reliability method (FORM) in probability theory, which means the minimum distance from the origin to the failure hypersurface, namely, the distance from the origin to the most probable point (MPP). Obviously, the unbearable computational cost caused by the nested double loop structure is still the key problem that needs to be solved. Therefore, inspired by SORA, an efficient decoupled strategy handling the deterministic optimization and non-probabilistic reliability analysis sequentially for NRBDO convex models was developed by Meng et al. [35]. For another, Jiang et al. [36] employed a satisfaction degree of interval to deal with the uncertain constraints. The satisfaction degree which represents the possibility that one interval is smaller than another is derived from an order-relation of interval numbers [37]. This kind of interval reliability index provides a clear and definite assessment criterion for the structural safety with uncertain-butbounded parameters, where $R_{e}=0$ stands for absolute failure, $R_{e}=1$ stands for absolute safety, $0<R_{e}<1$ stands for the degree of safety ( $R_{e}$ denotes the non-probabilistic reliability). Furthermore, Wang [38] expanded this thought into multi-dimensional cases and proposed a measure of structural non-probabilistic inter-

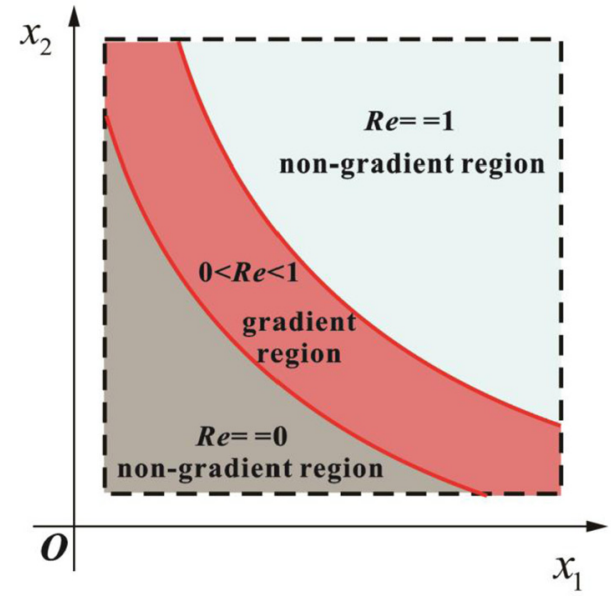

Fig. 1. Illustration of the design region in NRBDO.

val reliability based on the volume ratio theory, which provides a clear physical definition. However, as illustrated in Fig. 1, there exist non-gradient regions for this kind of reliability index in NRBDO problems, the traditional gradient-based optimization algorithms will be inapplicable. Under this circumstance, the global optimization algorithms should be an alternative, however, the calculation will sharply increase. To sum up, there are two major difficulties in dealing with RBDO under this non-probabilistic reliability index: (1) prohibitive computation caused by the nested optimization; (2) convergence difficulties caused by the non-gradient regions. Therefore, in order to address these problems, developing efficient solution strategies has become one of the most important issues that need to be resolved urgently.

Besides, it should be noticed that the non-probabilistic model has only been primitively applied to solving UMDO problems in the presence of uncertain-but-bounded parameters. Li et al. [39] proposed an uncertain multi-objective MDO methodology, which employs the interval model to represent the uncertain-butbounded parameters. Notwithstanding, compared with the probabilistic reliability based MDO problems, studies on RBMDO based on non-probabilistic theory are still rare at present, let alone the efficient methodology integrating MDO, uncertainty based multidisciplinary analysis (UMDA), non-probabilistic reliability assessment (NRA) and single loop strategy.

In view of all above statements, in this paper, a single loop approach is proposed to improve the efficiency of RBMDO for the multidisciplinary systems with interval parameters. Borrowing the decoupling idea, the proposed method develops an architecture where the MDO, UMDA, NRA and translating distance calculation (TDC) are performed in a sequential manner, and thus, the number of function calls are indeed reduced.

The reminder of the paper is structured as follows. First, the multidisciplinary system with interval uncertainty is briefly reviewed. Second, three uncertainty propagation analysis methods in multidisciplinary system are introduced, followed by the elaboration of reliability analysis in multidisciplinary system. Third, the single loop strategy is expounded, and the execution procedure is elaborated in detail. Fourth, one numerical example and one practical hypersonic wing dimensional design problem are conducted to demonstrate the validation of the proposed methods. Finally, this paper is closed with some conclusion remarks.

\section{Multidisciplinary system with interval uncertainties}

In this section, the introduction of general multidisciplinary system with interval uncertainties is conducted. A typical fourdiscipline system is taken as an example to clarify the issue and 


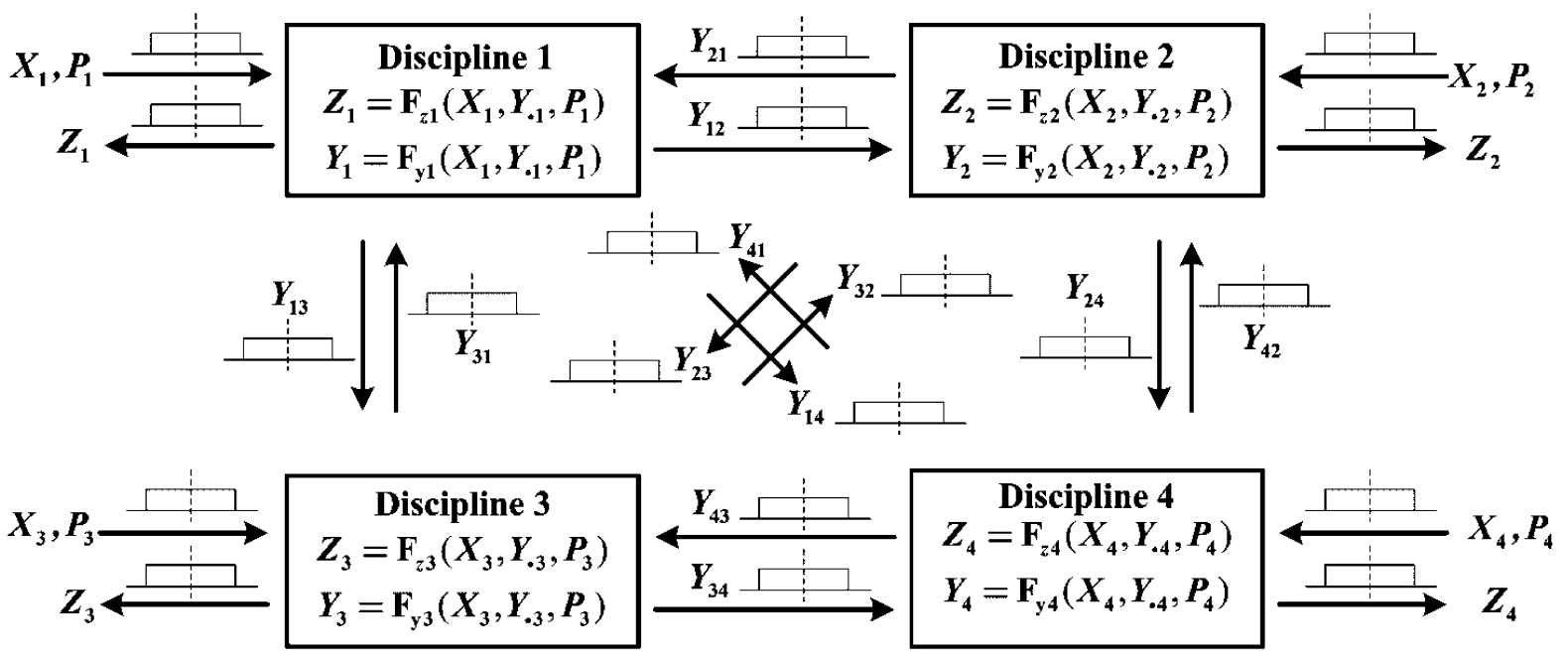

Fig. 2. Multidisciplinary system consisting of 4 coupled disciplines with interval uncertainties.

the interrelation in the multidisciplinary system is showed in Fig. 2, where each block represents a simulation program that belongs to a discipline. Furthermore, the notations utilized in the following content are explained as below.

Discipline, represents the basic module in the coupled multidisciplinary system. All the disciplines comprise the integrated multidisciplinary system by mutually exchanging the data and information. Moreover, the number of the disciplines is $n$.

$\boldsymbol{X}_{S}-$ System design variables, represents the design variables that are involved in more than one discipline.

$\boldsymbol{X}_{i}-$ Disciplinary design variables, represents the design variables that are only involved in discipline $i$.

Obviously, $\boldsymbol{X}_{s}$ and $\boldsymbol{X}_{i}$ are mutually exclusive sets that constitute the design variables. For example, with regards to the multidisciplinary optimization design of aircraft, in the aerodynamic disciplinary, the disciplinary design variables are the torsion angles of wing-root and wing-tip, etc. In the structure disciplinary, the disciplinary design variables are the dimensions of beams and ribs, the thickness of skin and so on. Furthermore, the system design variables refer to the span-chord ratio, the taper ratio, the wing area, etc. Note that, considering machining error, assembly variation, etc., in real projects, it is reasonable to treat the design variables as uncertainties as well.

$\boldsymbol{P}_{S}$-System uncertain parameters, represents the uncertain parameters that are involved in more than one discipline.

$\boldsymbol{P}_{i}$-Disciplinary uncertain parameters, represents the uncertain parameters only involved in discipline $i$.

Similarly, $\boldsymbol{P}_{s}$ and $\boldsymbol{P}_{i}$ are mutually exclusive sets that make up the input uncertain variables. The disciplinary uncertain parameters include the environmental parameter in the aerodynamic disciplinary, material property parameters in the structure disciplinary and so on. The system uncertain parameters include the load uncertainty, geometry dimension uncertainty, etc.

$\boldsymbol{Y}_{i j}(i \neq j)$-Linking variables, which are those functional outputs calculated in discipline $i$, at the same time, are required as inputs to discipline $j$, such as the surface pressure distribution in the aerodynamic disciplinary and surface deformation in the structure disciplinary.

$Z_{i}$-System outputs, which represent outputs of discipline $i$, such as lift coefficient, drag coefficient, pitch moment coefficient and lift-drag-ratio in the aerodynamic disciplinary, structure stress and strain in the structure disciplinary.

For simplification, we denote $\boldsymbol{Y}_{i}=\left\{\boldsymbol{Y}_{i j} \mid j=1, \ldots, n ; j \neq i\right\}$ as the set of linking variables generated as outputs from discipline $i$ and taken as inputs to the other disciplines and $\boldsymbol{Y}_{\bullet i}=$
$\left\{\boldsymbol{Y}_{1, i}, \ldots, \boldsymbol{Y}_{i-1, i}, \boldsymbol{Y}_{i+1, i}, \ldots, \boldsymbol{Y}_{n, i}\right\}$ as the set of linking variables generated as outputs from each of the discipline except discipline $i$ and taken as inputs to discipline $i$.

For discipline $i$, based on the discipline simulation model $\mathbf{F}_{\mathbf{y} i}(\bullet)$, the linking variables can be derived as:

$\boldsymbol{Y}_{i}=\mathbf{F}_{\mathbf{y i}}\left(\boldsymbol{X}_{s}, \boldsymbol{X}_{i}, \boldsymbol{Y}_{\bullet i}, \boldsymbol{P}_{s}, \boldsymbol{P}_{i}\right)$

Equally as well, the general output of discipline $i, Z_{i}$ ( $i=$ $1, \ldots, n)$, can be derived as:

$\boldsymbol{Z}_{i}=\mathbf{F}_{\mathbf{z} i}\left(\boldsymbol{X}_{s}, \boldsymbol{X}_{i}, \boldsymbol{Y}_{\bullet i}, \boldsymbol{P}_{s}, \boldsymbol{P}_{i}\right)$

\section{Reliability analysis in multidisciplinary system with interval uncertainties}

As the key stages of NRBMDO, uncertainty analysis methods as well as reliability assessment approaches in multidisciplinary system are discussed in this section.

\subsection{Uncertainty propagation analysis in multidisciplinary system}

Three multidisciplinary uncertainty analysis methods i.e. the first order interval Taylor series expansion method, the interval vertex approach and the direct optimization theorem are studied in this section.

\subsubsection{First order interval Taylor expansion method based on global sensitivity equation}

It is well known that interval Taylor series expansion is one of the most common methods to deal with the interval uncertainty propagation analysis for single discipline [40,41]. When the outputs are either linear or nearly linear with the inputs in the discipline, the first-order Taylor expansion method is a good choice to obtain the bounds of the outputs with high efficiency and accuracy. Based on this, the first-order Taylor expansion method is further studied to apply in multidisciplinary system.

In order to express the method more clearly, all the involved uncertainties are divided into two categories, namely, system uncertainties $\boldsymbol{U}_{s}$ and disciplinary uncertainties $\boldsymbol{U}_{d_{\_} i}(i=1, \ldots, n)$. The expressions of $\boldsymbol{U}_{s}$ and $\boldsymbol{U}_{d_{-} i}$ are formulated as follows:

$$
\boldsymbol{U}_{s}=\boldsymbol{X}_{s} \cup \boldsymbol{P}_{s}, \quad \boldsymbol{U}_{d \_i}=\boldsymbol{X}_{i} \cup \boldsymbol{P}_{i} \quad(i=1, \ldots, n)
$$

For convenience, uncertain parameters can be described by the following expressions. 


$$
\left\{\begin{array}{l}
\boldsymbol{U}_{s}^{c}=\frac{\overline{\boldsymbol{U}_{s}}+\underline{\boldsymbol{U}_{s}}}{2}, \quad \boldsymbol{U}_{s}^{r}=\frac{\overline{\boldsymbol{U}_{s}}-\underline{\boldsymbol{U}_{s}}}{2}, \\
\underline{\boldsymbol{U}_{s}}=\boldsymbol{U}_{s}^{c}-\boldsymbol{U}_{s}^{r}, \quad \overline{\boldsymbol{U}_{s}}=\boldsymbol{U}_{s}^{c}+\boldsymbol{U}_{s}^{r} \\
\boldsymbol{U}_{d_{-} i}^{c}=\frac{\overline{\boldsymbol{U}_{d \_i}}+\underline{\boldsymbol{U}_{d_{\_} i}}}{2}, \quad \boldsymbol{U}_{d_{-} i}^{r}=\frac{\overline{\boldsymbol{U}_{d_{-} i}}-\underline{\boldsymbol{U}_{d_{-} i}}}{2}, \\
\underline{\boldsymbol{U}_{d_{\_} i}}=\boldsymbol{U}_{d_{-} i}^{c}-\boldsymbol{U}_{d_{-} i}^{r}, \quad \overline{\boldsymbol{U}_{d_{-} i}}=\boldsymbol{U}_{d_{-} i}^{c}+\boldsymbol{U}_{d_{-} i}^{r}
\end{array}\right.
$$

where $\boldsymbol{U}_{s}^{c}$ and $\boldsymbol{U}_{d i}^{c}$ are the median values of $\boldsymbol{U}_{s}$ and $\boldsymbol{U}_{d_{-} i} ; \boldsymbol{U}_{s}^{r}$ and $\boldsymbol{U}_{d_{-} i}^{r}$ are the interval radius values; $\underline{\boldsymbol{U}_{s}}$ and $\underline{\boldsymbol{U}_{d_{\_} i}}$ are the lower bounds; $\overline{\boldsymbol{U}_{s}}$ and $\overline{\boldsymbol{U}_{d_{-} i}}$ are the upper bounds. Note that, in the whole paper, the following rules of symbols are utilized. The superscript " $r$ " means the radius value, the superscript " $c$ " means the median value, the overline "-" stands for the upper bound, the underline "-" stands for the lower bound.

Then, rewrite Eq. (1) and Eq. (2) as

$$
\begin{aligned}
& \boldsymbol{Y}_{i}=\mathbf{F}_{\mathbf{y} i}\left(\boldsymbol{U}_{s}, \boldsymbol{U}_{d_{\_} i}, \boldsymbol{Y}_{\bullet i}\right) \\
& \boldsymbol{Z}_{i}=\mathbf{F}_{\mathbf{z i}}\left(\boldsymbol{U}_{s}, \boldsymbol{U}_{d_{-} i}, \boldsymbol{Y}_{\bullet i}\right)
\end{aligned}
$$

Enlightened by the derivation process of the well-known global sensitivity equation (GSE) [42], we can approximate $\boldsymbol{Y}_{i}$ at the median value of $\boldsymbol{U}_{s}, \boldsymbol{U}_{d_{-} i}$ and $\boldsymbol{Y}_{\bullet i}$ by utilizing the first order Taylor expansion method [43]:

$$
\begin{aligned}
\boldsymbol{Y}_{i}= & \boldsymbol{Y}_{i}^{c}+\frac{\partial \mathbf{F}_{\mathbf{y} i}}{\partial \boldsymbol{U}_{s}}\left(\boldsymbol{U}_{s}-\boldsymbol{U}_{s}^{c}\right)+\frac{\partial \mathbf{F}_{\mathbf{y} i}}{\partial \boldsymbol{U}_{d_{-} i}}\left(\boldsymbol{U}_{d_{-} i}-\boldsymbol{U}_{d_{-} i}^{c}\right) \\
& +\frac{\partial \mathbf{F}_{\mathbf{y} i}}{\partial \boldsymbol{Y}_{\bullet i}}\left(\boldsymbol{Y}_{\bullet i}-\boldsymbol{Y}_{\bullet i}^{c}\right) \\
= & \boldsymbol{Y}_{i}^{c}+\frac{\partial \mathbf{F}_{\mathbf{y} i}}{\partial \boldsymbol{U}_{s}}\left(\boldsymbol{U}_{s}-\boldsymbol{U}_{s}^{c}\right)+\frac{\partial \mathbf{F}_{\mathbf{y} i}}{\partial \boldsymbol{U}_{d_{-} i}}\left(\boldsymbol{U}_{d_{-} i}-\boldsymbol{U}_{d_{-} i}^{c}\right) \\
& +\sum_{\substack{j=1 \\
j \neq i}}^{n} \frac{\partial \mathbf{F}_{\mathbf{y} i}}{\partial \boldsymbol{Y}_{j, i}}\left(\boldsymbol{Y}_{j, i}-\boldsymbol{Y}_{j, i}^{c}\right)
\end{aligned}
$$

Obviously, the median values of $\boldsymbol{Y}_{i}$ can be acquired by one time of multidisciplinary analysis:

$$
\boldsymbol{Y}_{i}^{c}=\mathbf{F}_{\mathbf{y} i}\left(\boldsymbol{U}_{s}^{c}, \boldsymbol{U}_{d \_i}^{c}, \boldsymbol{Y}_{\bullet i}^{c}\right)
$$

Based on the interval arithmetic, the upper and lower bounds of $\boldsymbol{Y}_{i}$ can be obtained:

$$
\begin{aligned}
\overline{\boldsymbol{Y}_{i}}= & \boldsymbol{Y}_{i}^{c}+\left|\frac{\partial \mathbf{F}_{\mathbf{y} i}}{\partial \boldsymbol{U}_{s}}\right|\left(\overline{\boldsymbol{U}_{s}}-\boldsymbol{U}_{s}^{c}\right)+\left|\frac{\partial \mathbf{F}_{\mathbf{y} i}}{\partial \boldsymbol{U}_{d_{-} i}}\right|\left(\overline{\boldsymbol{U}_{d_{-} i}}-\boldsymbol{U}_{d_{\_} i}^{c}\right) \\
& +\sum_{\substack{j=1 \\
j \neq i}}^{n}\left|\frac{\partial \mathbf{F}_{\mathbf{y} i}}{\partial \boldsymbol{Y}_{j, i}}\right|\left(\overline{\boldsymbol{Y}_{j, i}}-\boldsymbol{Y}_{j, i}^{c}\right) \\
\underline{\boldsymbol{Y}}_{i}= & \boldsymbol{Y}_{i}^{c}+\left|\frac{\partial \mathbf{F}_{\mathbf{y} i}}{\partial \boldsymbol{U}_{s}}\right|\left(\overline{\boldsymbol{U}_{s}}-\boldsymbol{U}_{s}^{c}\right)-\left|\frac{\partial \mathbf{F}_{\mathbf{y} i}}{\partial \boldsymbol{U}_{d_{-} i}}\right|\left(\overline{\boldsymbol{U}_{d_{-} i}}-\boldsymbol{U}_{d_{-} i}^{c}\right) \\
& -\sum_{\substack{j=1 \\
j \neq i}}^{n}\left|\frac{\partial \mathbf{F}_{\mathbf{y} i}}{\partial \boldsymbol{Y}_{j, i}}\right|\left(\overline{\boldsymbol{Y}_{j, i}}-\boldsymbol{Y}_{j, i}^{c}\right)
\end{aligned}
$$
by

Then, the radii of the coupled state variables can be deduced

$$
\boldsymbol{Y}_{i}^{r}=\left|\frac{\partial \mathbf{F}_{\mathbf{y} i}}{\partial \boldsymbol{U}_{s}}\right| \boldsymbol{U}_{s}^{r}+\left|\frac{\partial \mathbf{F}_{\mathbf{y} i}}{\partial \boldsymbol{U}_{d_{-} i}}\right| \boldsymbol{U}_{d_{-} i}^{r}+\sum_{\substack{j=1 \\ j \neq i}}^{n}\left|\frac{\partial \mathbf{F}_{\mathbf{y} i}}{\partial \boldsymbol{Y}_{j, i}}\right| \Delta \boldsymbol{Y}_{j, i}^{r}
$$

Rewrite Eq. (10) with matrix form as follows:

$$
\begin{aligned}
& {\left[\begin{array}{cccc}
\mathbf{I}_{\mathbf{1}} & -\left|\frac{\partial \mathbf{F}_{\mathbf{y} 1}}{\partial \mathbf{Y}_{21}}\right| & \ldots & -\left|\frac{\partial \mathbf{F}_{\mathbf{y} 1}}{\partial \mathbf{Y}_{n 1}}\right| \\
-\left|\frac{\partial \mathbf{F}_{\mathbf{y} 2}}{\partial \mathbf{Y}_{12}}\right| & \mathbf{I}_{\mathbf{2}} & \ldots & -\left|\frac{\partial \mathbf{F}_{\mathbf{y} 2}}{\partial \boldsymbol{Y}_{n 2}}\right| \\
\vdots & \vdots & \ddots & \vdots \\
-\left|\frac{\partial \mathbf{F}_{\mathbf{y} n}}{\partial \mathbf{Y}_{1 n}}\right| & -\left|\frac{\partial \mathbf{F}_{\mathbf{y} n}}{\partial \mathbf{Y}_{2 n}}\right| & \ldots & \mathbf{I}_{n}
\end{array}\right]\left[\begin{array}{c}
\boldsymbol{Y}_{1}^{r} \\
\boldsymbol{Y}_{2}^{r} \\
\vdots \\
\boldsymbol{Y}_{n}^{r}
\end{array}\right]} \\
& =\left[\begin{array}{c}
\left|\frac{\partial \mathbf{F}_{\mathbf{y} 1}}{\partial \boldsymbol{U}_{s}}\right| \\
\left|\frac{\partial \mathbf{F}_{\mathbf{y} 2}}{\partial \boldsymbol{U}_{s}}\right| \\
\vdots \\
\left|\frac{\partial \mathbf{F}_{\mathbf{y n}}}{\partial \boldsymbol{U}_{s}}\right|
\end{array}\right] \boldsymbol{U}_{S}^{r}+\operatorname{diag}\left[\begin{array}{c}
\left|\frac{\partial \mathbf{F}_{\mathbf{y} 1}}{\partial \boldsymbol{U}_{d \_}}\right| \\
\left|\frac{\partial \mathbf{F}_{\mathbf{y} 2}}{\partial \boldsymbol{U}_{d \_i}}\right| \\
\vdots \\
\left|\frac{\partial \mathbf{F}_{\mathbf{y} n}}{\partial \boldsymbol{U}_{d \_} i}\right|
\end{array}\right] \boldsymbol{U}_{d_{-} i}^{r}
\end{aligned}
$$

where "diag" means the diagonal matrix.

Similarly, $\boldsymbol{Z}_{i}$ can be approximated as

$$
\begin{aligned}
\boldsymbol{Z}_{i}= & \boldsymbol{Z}_{i}^{c}+\frac{\partial \mathbf{F}_{z i}}{\partial \boldsymbol{U}_{s}}\left(\boldsymbol{U}_{s}-\boldsymbol{U}_{s}^{c}\right)+\frac{\partial \mathbf{F}_{z i}}{\partial \boldsymbol{U}_{d_{-} i}}\left(\boldsymbol{U}_{d_{-} i}-\boldsymbol{U}_{d_{\_} i}^{c}\right) \\
& +\sum_{\substack{j=1 \\
j \neq i}}^{n} \frac{\partial \mathbf{F}_{z i}}{\partial \boldsymbol{Y}_{j, i}}\left(\boldsymbol{Y}_{j, i}-\boldsymbol{Y}_{j, i}^{c}\right)
\end{aligned}
$$

where $\boldsymbol{Z}_{i}^{c}=\mathbf{F}_{z i}\left(\boldsymbol{U}_{s}^{c}, \boldsymbol{U}_{d_{-} i}^{c}, \boldsymbol{Y}_{\bullet i}^{c}\right)$.

And then, the upper and lower bounds of $\boldsymbol{Z}_{i}$ can be calculated as

$$
\begin{aligned}
\overline{\boldsymbol{Z}_{i}}= & \boldsymbol{Z}_{i}^{c}+\left|\frac{\partial \mathbf{F}_{z i}}{\partial \boldsymbol{U}_{s}}\right|\left(\overline{\boldsymbol{U}_{s}}-\boldsymbol{U}_{s}^{c}\right)+\left|\frac{\partial \mathbf{F}_{z i}}{\partial \boldsymbol{U}_{d_{-} i}}\right|\left(\overline{\boldsymbol{U}_{d_{-} i}}-\boldsymbol{U}_{d_{-} i}^{c}\right) \\
& +\sum_{\substack{j=1 \\
j \neq i}}^{n}\left|\frac{\partial \mathbf{F}_{z i}}{\partial \boldsymbol{Y}_{j, i}}\right|\left(\overline{\boldsymbol{Y}_{j, i}}-\boldsymbol{Y}_{j, i}^{c}\right) \\
\underline{\boldsymbol{Z}}_{i}= & \boldsymbol{Z}_{i}^{c}+\left|\frac{\partial \mathbf{F}_{z i}}{\partial \boldsymbol{U}_{s}}\right|\left(\overline{\boldsymbol{U}_{s}}-\boldsymbol{U}_{s}^{c}\right)-\left|\frac{\partial \mathbf{F}_{z i}}{\partial \boldsymbol{U}_{d_{-} i}}\right|\left(\overline{\boldsymbol{U}_{d_{-} i}}-\boldsymbol{U}_{d_{-} i}^{c}\right) \\
& -\sum_{\substack{j=1 \\
j \neq i}}^{n}\left|\frac{\partial \mathbf{F}_{z i}}{\partial \boldsymbol{Y}_{j, i}}\right|\left(\overline{\boldsymbol{Y}_{j, i}}-\boldsymbol{Y}_{j, i}^{c}\right)
\end{aligned}
$$

Then, the radii of the coupled state variables can be deduced by

$$
\boldsymbol{Z}_{i}^{r}=\left|\frac{\partial \mathbf{F}_{z i}}{\partial \boldsymbol{U}_{s}}\right| \boldsymbol{U}_{s}^{r}+\left|\frac{\partial \mathbf{F}_{z i}}{\partial \boldsymbol{U}_{d_{-} i}}\right| \boldsymbol{U}_{d_{-} i}^{r}+\sum_{\substack{j=1 \\ j \neq i}}^{n}\left|\frac{\partial \mathbf{F}_{z i}}{\partial \boldsymbol{Y}_{j, i}}\right| \Delta \boldsymbol{Y}_{j, i}^{r}
$$

Rewrite Eq. (14) with matrix form as follows:

$$
\begin{aligned}
{\left[\begin{array}{c}
\boldsymbol{Z}_{1}^{r} \\
\boldsymbol{Z}_{2}^{r} \\
\vdots \\
\boldsymbol{Z}_{n}^{r}
\end{array}\right]=} & {\left[\begin{array}{cccc}
\mathbf{0} & \left|\frac{\partial \mathbf{F}_{z 1}}{\partial \boldsymbol{Y}_{21}}\right| & \ldots & \left|\frac{\partial \mathbf{F}_{z 1}}{\partial \mathbf{Y}_{n 1}}\right| \\
\left|\frac{\partial \mathbf{F}_{z 2}}{\partial \mathbf{Y}_{12}}\right| & \mathbf{0} & \ldots & \left|\frac{\partial \mathbf{F}_{z 2}}{\partial \mathbf{Y}_{n 2}}\right| \\
\vdots & \vdots & \ddots & \vdots \\
\left|\frac{\partial \mathbf{F}_{z n}}{\partial \boldsymbol{Y}_{1 n}}\right| & \left|\frac{\partial \mathbf{F}_{z n}}{\partial \boldsymbol{Y}_{2 n}}\right| & \ldots & \mathbf{0}
\end{array}\right]\left[\begin{array}{c}
\boldsymbol{Y}_{1}^{r} \\
\boldsymbol{Y}_{2}^{r} \\
\vdots \\
\boldsymbol{Y}_{n}^{r}
\end{array}\right] } \\
& +\left[\begin{array}{c}
\left|\frac{\partial \mathbf{F}_{z 1}}{\partial \boldsymbol{U}_{s}}\right| \\
\left|\frac{\partial \mathbf{F}_{z 2}}{\partial \boldsymbol{U}_{s}}\right| \\
\vdots \\
\left|\frac{\partial \mathbf{F}_{z n}}{\partial \boldsymbol{U}_{s}}\right|
\end{array}\right] \boldsymbol{U}_{s}^{r}+\operatorname{diag}\left[\begin{array}{c}
\left|\frac{\partial \mathbf{F}_{z 1}}{\partial \mathbf{U}_{d_{-} i}}\right| \\
\left|\frac{\partial \mathbf{F}_{z 2}}{\partial \mathbf{U}_{d_{-} i}}\right| \\
\vdots \\
\\
\left|\frac{\partial \mathbf{F}_{z n}}{\partial \mathbf{U}_{d_{-} i}}\right|
\end{array}\right] \boldsymbol{U}_{d_{-} i}^{r}
\end{aligned}
$$


Consequently, the radii of $\boldsymbol{Y}_{i}$ and $\boldsymbol{Z}_{i}$ can be obtained by solving Eq. (11) and Eq. (15) with matrix operations. Thus the bounds of the linking variables and outputs can be expressed as

$\left\{\begin{array}{l}\overline{\boldsymbol{Y}_{i}}=\boldsymbol{Y}_{i}^{c}+\boldsymbol{Y}_{i}^{r} \\ \underline{\boldsymbol{Y}}_{i}=\boldsymbol{Y}_{i}^{c}-\boldsymbol{Y}_{i}^{r}\end{array} \quad\left\{\begin{array}{l}\overline{\boldsymbol{Z}_{i}}=\boldsymbol{Z}_{i}^{c}+\boldsymbol{Z}_{i}^{r} \\ \underline{\boldsymbol{Z}}_{i}=Z_{i}^{c}-\boldsymbol{Z}_{i}^{r}\end{array}\right.\right.$

\subsubsection{Interval vertex theorem}

As stated in Ref. [44], based on the extreme theorem in convex analysis, if the output is a convex (or concave) function of the inputs, the extreme values of the output will reached on the boundary vertices. Under this circumstance, from the interval mathematical view of point, the interval solution, computed by the vertex solution theorem, is exact. Therefore, when the linking variables $\boldsymbol{Y}_{i}$ and system outputs $\boldsymbol{Z}_{i}$ are either convex or concave to the input uncertainties $\boldsymbol{X}_{s}, \boldsymbol{P}_{s}, \boldsymbol{X}_{i}, \boldsymbol{P}_{i}$ in the multidisciplinary, the interval vertex theorem can be utilized to obtain the accurate interval solution. The implementation procedure can be organized as:

(1) Obtain the input vector of uncertain parameters, which can be mathematically written as

$\boldsymbol{U}=\boldsymbol{X}_{s} \cup \boldsymbol{P}_{s} \cup\left(\boldsymbol{X}_{1} \cup \ldots \cup \boldsymbol{X}_{n}\right) \cup\left(\boldsymbol{P}_{1} \cup \ldots \cup \boldsymbol{P}_{n}\right)$

where the number of elements in $\boldsymbol{U}$ denotes $N_{\text {total }}$ and rewrite Eq. (17) as:

$$
\boldsymbol{U}=\left[\begin{array}{llll}
\boldsymbol{U}(1) & \boldsymbol{U}(2) & \ldots & \boldsymbol{U}\left(N_{\text {total }}\right)
\end{array}\right]
$$

(2) Combine the vertexes of the input parameters as the new set of certain input parameters through constructing the interval vertex array $\boldsymbol{M}_{\text {vertex }}$ shown in Eq. (19), which can be easily accomplished by use of self-complied programs.

$$
\begin{aligned}
\boldsymbol{M}_{\text {vertex }}= & {\left[\begin{array}{ccc}
1+\beta_{1} & \cdots & 1+\beta_{N_{\text {total }}} \\
\vdots & 1+\beta_{j}(-1)^{<(i-1) / 2^{N_{\text {total }}-j}>} & \vdots \\
1-\beta_{1} & \cdots & 1-\beta_{N_{\text {total }}}
\end{array}\right] } \\
& (<*>\text { represents integer less than } * .)
\end{aligned}
$$

where $i$ and $j$ represent the array row number and column umber as well as $\max i=2^{N_{\text {total }}}$ and $\max j=N_{\text {total }}$. Each of the elements in $\boldsymbol{\beta}=\left[\beta_{1}, \beta_{2}, \ldots, \beta_{N_{\text {total }}}\right]$ corresponds to the deviation factor of the elements in input vector $\boldsymbol{U}$. And the deviation factor is defined as the ratio of the radius to the median value for uncertain parameter.

(3) Then, the corresponding input vector $\boldsymbol{U}_{V}$ can be calculated through Eq. (20).

$\boldsymbol{U}_{V}=\boldsymbol{U}_{\text {Vertex }} \boldsymbol{M}_{\text {Vertex }}$

where $\boldsymbol{U}_{\text {Vertex }}=\operatorname{diag}\left(\boldsymbol{U}(1), \boldsymbol{U}(2), \ldots, \boldsymbol{U}\left(N_{\text {total }}\right)\right)$

(4) Based on $\boldsymbol{U}_{V}$, calculate the outputs set through multidisciplinary analysis as follows:

$\left\{\begin{array}{l}\boldsymbol{Y}_{\text {set }}=\mathbf{F}_{\mathbf{y} i}\left(\boldsymbol{U}_{V}, \boldsymbol{Y}_{\bullet i}\right) \\ \boldsymbol{Z}_{\text {set }}=\mathbf{F}_{z i}\left(\boldsymbol{U}_{V}, \boldsymbol{Y}_{\bullet i}\right)\end{array}\right.$

Obviously, the number of multidisciplinary analyses is $2^{N_{\text {total }}}$.

(5) Eventually, the maximum and minimum values in the output set should be selected as the upper and low bounds of the multidisciplinary outputs.

$$
\left\{\begin{array} { l } 
{ \overline { \boldsymbol { Y } } _ { i } = \operatorname { m a x } ( \boldsymbol { Y } _ { \text { set } } ) } \\
{ \underline { \boldsymbol { Y } } _ { i } = \operatorname { m i n } ( \boldsymbol { Y } _ { \text { set } } ) }
\end{array} \quad \left\{\begin{array}{l}
\overline{\boldsymbol{Z}_{i}}=\max \left(\boldsymbol{Z}_{\text {set }}\right) \\
\underline{\boldsymbol{Z}_{i}}=\min \left(\boldsymbol{Z}_{\text {set }}\right)
\end{array}\right.\right.
$$

\subsubsection{Direct optimization approach}

It is widely acknowledged that interval analysis is essentially an optimization issue. Based on this, in the section, the uncertainty propagation in multidisciplinary system are modeled as a deterministic multidisciplinary optimization problem as follows:

$$
\begin{aligned}
& \left\{\begin{array}{l}
\text { find } \boldsymbol{X}_{s}, \boldsymbol{P}_{s}, \boldsymbol{X}_{i}, \boldsymbol{P}_{i} \\
\min / \max \boldsymbol{Y}_{i}, \boldsymbol{Z}_{i} \\
\text { s.t. } \quad \boldsymbol{Y}_{i}=\mathbf{F}_{\mathbf{y} i}\left(\boldsymbol{X}_{s}, \boldsymbol{X}_{i}, \boldsymbol{Y}_{\bullet i}, \boldsymbol{P}_{s}, \boldsymbol{P}_{i}\right) \\
\quad \boldsymbol{Z}_{i}=\mathbf{F}_{\mathbf{z i}}\left(\boldsymbol{X}_{s}, \boldsymbol{X}_{i}, \boldsymbol{Y}_{\bullet i}, \boldsymbol{P}_{s}, \boldsymbol{P}_{i}\right) \\
\quad \boldsymbol{X}_{s} \in\left[\underline{\boldsymbol{X}_{s}}, \overline{\boldsymbol{X}_{s}}\right], \boldsymbol{X}_{i} \in\left[\underline{\boldsymbol{X}_{i}}, \overline{\boldsymbol{X}_{i}}\right], \boldsymbol{P}_{i} \in\left[\underline{\boldsymbol{P}_{i}}, \overline{\boldsymbol{P}_{i}}\right], \boldsymbol{P}_{s} \in\left[\underline{\boldsymbol{P}_{s}}, \overline{\boldsymbol{P}_{s}}\right]
\end{array}\right. \\
& (i=1, \ldots, n)
\end{aligned}
$$

Apparently, solving Eq. (23) with existing optimization algorithms is a feasible way to acquire the exact bounds of outputs. On the one hand, when the optimization problem is continuous and unimodal, the sensitivity-based optimization methods, i.e., the feasible direction method, the deepest descent method, the sequential quadratic programming and so on, are feasible and efficient. On the other hand, when the amount of the input variables is large and the gradient information is difficult to obtain, which is really common in practical engineering, the non-gradient optimization methods, namely, the downhill simplex method, the direct search method, the genetic algorithm, the particle swarm optimization algorithm etc., are introduced to address this issue.

\subsubsection{Hybrid approach based on the concurrent strategy}

Based on the above analysis, we can see that solving the bounds of linking variables and system outputs are independent when the interval vertex theorem and the direct optimization approach are applied. That is to say, the interval bounds of any single output in multidisciplinary system can be solved by selecting either the interval vertex theorem or the direct optimization approach in the same case. And obviously, the concurrent strategy can be utilized to improve the efficiency under this circumstance. Therefore, a hybrid approach, where both the interval vertex theorem and the direct optimization approach are utilized, can be proposed. For the implementation of the hybrid approach, we firstly separate the outputs, including the linking variables and the system outputs into two categories according to the application conditions of the interval vertex theorem and the direct optimization approach. Then, all the solving processes corresponding to the interval vertex theorem and the direct optimization approach are conducted in parallel, which can greatly reduce the computational time. The specified implement procedure can be illustrated in Fig. 3.

\subsubsection{Discussions on the four methods}

In order to provide general guidelines for choosing the more appropriate technique among the four methods, the comparisons of all the four methods are conducted on computational amount and accuracy.

(1) As stated in section 3.1.1, the first order interval Taylor expansion method is an approximation method that possesses high efficiency. However, this method is only suitable for the linear and nearly linear situations.

(2) In actual engineering, the multidisciplinary systems are always highly nonlinear and the first order interval Taylor expansion method is no longer fit here. In this case, the later three methods, which can provide exact solutions in theory, are supposed to be utilized. Considering that the computational accuracy is the same for all the three method, the computational amount of the three methods is compared to choose the most appropriate one. 


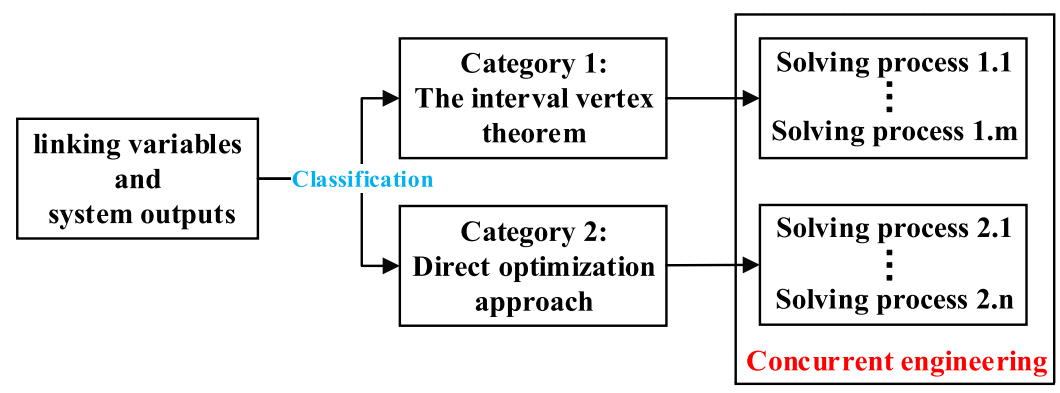

Fig. 3. Procedure of the hybrid approach.

(A) For the interval vertex theorem, as explained in section 3.1.2, the number of multidisciplinary analysis (MDA) is $N_{\text {interval }}=2^{N_{\text {total }} \text {. }}$

(B) For the director optimization approach, denote the number of MDA involved in optimization procedure for any uncertain parameters is $n_{0 i}\left(i=1, \ldots, N_{\text {total }}\right)$, then the number of MDA in the whole solving process can be written as

$$
N_{\text {optimization }}=\sum_{i=N_{\text {total }}} n_{0_{-} i}
$$

(C) For the hybrid approach, the numbers of uncertain parameters in category I and II are denote as $N_{\text {total_I }}$ and $N_{\text {total_II }}$ respectively. Then, the number of MDA in this methods can be expressed as

$$
N_{\text {hybrid }}=2^{N_{\text {total }} 1}+\sum_{i=N_{\text {total_2 }}} n_{0_{-} i}
$$

Obviously, we can easily choose the best method by comparing $N_{\text {interval }}, N_{\text {optimization }}$ and $N_{\text {hybrid }}$.

To sum up, each of the four methods has its own merits, thus in actual engineering projects, we are supposed to choose which method to be utilized in light of actual situations.

\subsection{Non-probabilistic reliability analysis in multidisciplinary system}

As claimed in Ref. [29], when evaluating the non-probabilistic reliability for structures, the non-probabilistic set-theoretic stressstrength interference model can give a clear and transparent explanation for the state of uncertain stress and strength. In this paper, we expand this concept into multidisciplinary field so as to provide an appropriate non-probabilistic safety measure for multidisciplinary system.

First, the limit-state functions of multidisciplinary can be formulated as

$\boldsymbol{G}_{i}=\boldsymbol{Z}_{i}-\boldsymbol{Z}_{i}^{0} \quad(i=1,2, \ldots, n)$

where $\boldsymbol{G}_{i}$ denotes the limit-state function vector of discipline $i, \boldsymbol{Z}_{i}$ and $Z_{i}^{0}$ respectively denotes the actual and allowable multidisciplinary responses of discipline $i$.

It is well known that $\boldsymbol{Z}_{i}$ and $\boldsymbol{Z}_{i}^{0}$ are influenced by many uncertain factors in practical engineering projects. Take the fluidstructure coupling optimization design of aircraft design, for example. The dynamic and static mechanical responses, including the stress level, displacement, fatigue life, frequency, etc., are always the major concerns in aircraft design. Distinctly, all the mechanical responses rely on many factors, including aerodynamic loads, material properties, geometry size and so on. Besides, the following facts should be noted: (1) For the aerodynamic loads, the variability will be triggered by the perturbation of atmosphere parameters (temperature, humidity, viscidity, etc.) and the instability of flight state (the angle of attack, sideslip angle, etc.). (2) For the material properties, the inhomogeneity of microstructure and the flaw caused by machining are the main reasons that lead to the dispersion. (3) The machining tolerance and surface roughness in geometry size as well as the cognitive dissonance of designers caused by the lack of knowledge are indeed the uncertain sources that cannot be ignored.

Consequently, the responses are supposed to be recognized as uncertainties in engineering design. In this paper, considering the fact that the sample information about uncertain parameters is always poor in reality, especially for the complex engineering projects, the uncertain parameters are described as the basic nonprobabilistic intervals. Therefore, after propagation, the multidisciplinary responses are uncertain-but-bounded and the element of $\boldsymbol{Z}_{i}$ and $\boldsymbol{Z}_{i}^{0}$ can be expressed as

$\left\{\begin{array}{l}Z_{i, j} \in\left[\underline{Z_{i, j}}, \overline{Z_{i, j}}\right] \\ Z_{i, j}^{0} \in\left[\underline{Z_{i, j}^{0}}, \overline{Z_{i, j}^{0}}\right]\end{array} \quad i=1,2, \ldots, n ; j=1,2, \ldots, m_{i}\right.$

where $n$ represents the number of disciplines and $m_{i}$ represents the number of the limit-state functions involved in disciplinary $i$. Obviously, the total number of the limit-state functions is $N_{t}=$ $\sum_{i=1}^{n} m_{i}$.

As shown in Eq. (26), the value of the limit-state function can be utilized to judge the system state. For some types of constraints, such as stress and displacement, the positive value of the limit-state function indicates the failure state while the negative value indicates the safe state. On the contrary, for some other constraints, such as fatigue life and lift-drag ratio, the positive value means the safe state while the negative value means the failure state. In order to make the following analysis process clear, we unify the limit-state functions vector as

$$
\boldsymbol{M}=\boldsymbol{R}-\boldsymbol{R}^{\mathbf{0}}
$$

where $\boldsymbol{M}>\mathbf{0}$, the state of the system is safe, otherwise, the system is dangerous. Apparently, the dimensions of $\boldsymbol{M}$ is $N_{t}$.

Actually, with the purpose of evaluating the failure possibility of each limit-state function in multidisciplinary system, an appropriate index that implies that an interval number is better than another but not that one is larger than another is needed. In this paper, the non-probabilistic index proposed in [29] is adopted for its clearer physical implication to measure the safety degree of structures with interval parameters. And then, the measure of the structural safety can be defined as the area ratio of the safe region $S_{\text {safe }}$ to the whole region $S_{\text {total }}$. For generality, we define Eq. (27) as the typical limit-state function, and the mathematical expression of the reliability is described by Eq. (28) as well as the physical meaning illustrated by Fig. 4 . 
(1)

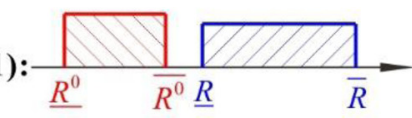

(2):

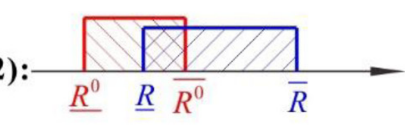

(3):
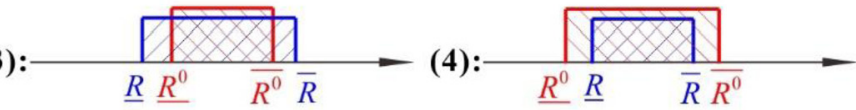

(5):

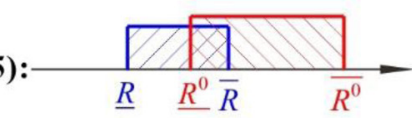

(a) one dimension coordinate system

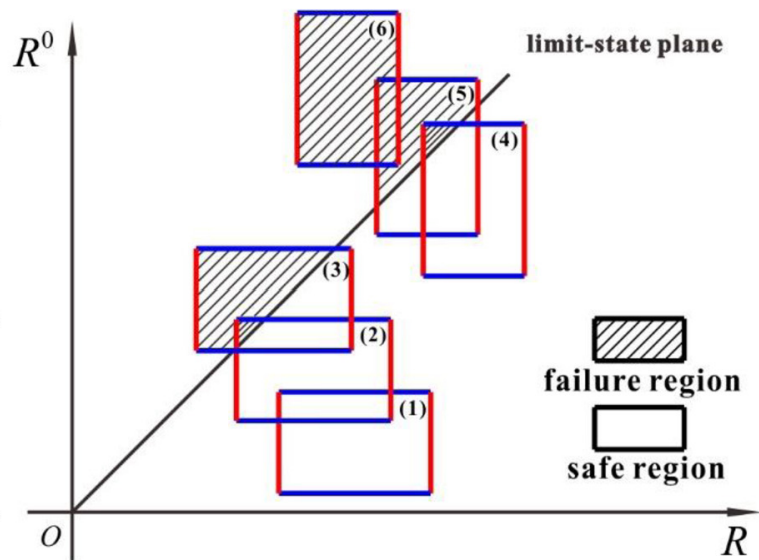

(b) two dimension coordinate system

Fig. 4. The six situations of the interval interference model.

$M=R-R^{0}$

$\eta(G>0)=\eta\left(R-R^{0}>0\right)=\frac{S_{\text {safe }}}{S_{\text {total }}}$

where $\eta(\bullet)$ denotes the non-probabilistic reliability.

Given the above understanding, the calculation of the reliability index is derived by considering the different locations of intervals. Because $R$ and $R^{0}$ are positive in actual engineering projects, all the six situations can be illustrated in Fig. 4(a) in one dimension coordinate system. In order to improve understandability and express the physical meaning more clearly, the situations shown in one dimension coordinate system are equivalently transformed in two dimension coordinate system in Fig. 4(b). Based on this, the corresponding mathematical expressions can be further derived and summarized in Eq. (29).

$\eta= \begin{cases}0 & \text { when: } \underline{R}<\bar{R} \leq \underline{R^{0}}<\overline{R^{0}} \\ \left(\bar{R}-\underline{R^{0}}\right)^{2} /\left(8 R^{0 r} R^{r}\right) & \text { when: } \overline{R^{0}}>\bar{R}>\underline{R^{0}}>\underline{R} \\ \left(R^{r}-R^{c}+R^{0 c}\right) /\left(2 R^{r}\right) & \text { when: } \overline{R^{0}}>\bar{R}>\underline{R}>\underline{R^{0}} \\ \left(R^{0 r}+R^{c}-R^{0 c}\right) /\left(2 R^{0 r}\right) & \text { when: } \bar{R}>\overline{R^{0}}>\underline{R^{0}}>\underline{R} \\ 1-\left(\overline{R^{0}}-\underline{R}\right)^{2} /\left(8 R^{r} R^{0 r}\right) & \text { when: } \bar{R}>\overline{R^{0}}>\underline{R}>\underline{R^{0}} \\ 1 & \text { when: } \bar{R}>\underline{R} \geq \overline{R^{0}}>\underline{R^{0}}\end{cases}$

\section{Decoupling approach for non-probabilistic reliability based multidisciplinary design optimization}

The focus of our efforts is to work out the NRBMDO problem more efficiently. In order to achieve the goal, in this section, the single loop strategy is introduced to solve the NRBMDO problem by conducting MDO, UMDA, NRA in single level.

\subsection{Non-probabilistic reliability index based multidisciplinary design optimization}

As stated above, the multidisciplinary system contains $n$ coupled disciplines, the design variables $\boldsymbol{X}_{s}$ and $\boldsymbol{X}_{i}(i=1, \ldots, n)$ as well as the model parameters $\boldsymbol{P}_{s}$ and $\boldsymbol{P}_{i}(i=1, \ldots, n)$ are uncertain. The general RBMDO problem can be expressed as

$$
\begin{cases}\text { find } & \boldsymbol{X}_{s}^{c}, \boldsymbol{X}_{i}^{c} \\ \min & f^{c}\left(\boldsymbol{X}_{s}, \boldsymbol{X}_{i}\right) \\ \text { s.t. } & \boldsymbol{\eta}\{\boldsymbol{M} \geq \mathbf{0}\} \geq \boldsymbol{\eta}_{\mathbf{0}} \\ & \boldsymbol{Y}_{i}=\mathbf{F}_{\mathbf{y} i}\left(\boldsymbol{X}_{s}, \boldsymbol{X}_{i}, \boldsymbol{Y}_{\bullet i}, \boldsymbol{P}_{s}, \boldsymbol{P}_{i}\right) \\ & \boldsymbol{Z}_{i}=\mathbf{F}_{\mathbf{z} i}\left(\boldsymbol{X}_{s}, \boldsymbol{X}_{i}, \boldsymbol{Y}_{\bullet i}, \boldsymbol{P}_{s}, \boldsymbol{P}_{i}\right) \\ & \boldsymbol{X}_{s}^{c} \in\left[\boldsymbol{X}_{s}^{L}, \boldsymbol{X}_{s}^{U}\right], \boldsymbol{X}_{i}^{c} \in\left[\boldsymbol{X}_{i}^{L}, \boldsymbol{X}_{i}^{U}\right]\end{cases}
$$

where $\boldsymbol{X}_{s}^{c}$ and $\boldsymbol{X}_{i}^{c}$ represent the media values of the uncertain design variables. $\boldsymbol{\eta}_{\mathbf{0}}$ is the allowable non-probabilistic reliability vector for the constraints. $f^{c}\left(\boldsymbol{X}_{s}, \boldsymbol{X}_{i}\right)$ is the nominal value of the objective. $\boldsymbol{X}_{s}^{L}$ and $\boldsymbol{X}_{s}^{U}$ are the lower and upper limits of $\boldsymbol{X}_{s}^{c} . \boldsymbol{X}_{i}^{L}$ and $\boldsymbol{X}_{i}^{U}$ are the lower and upper limits of $\boldsymbol{X}_{i}^{c}$, all these define the boundaries of design space. Additionally, the meaning of the other parameters can be found in section 2 and 3 .

Apparently, the conventional non-probabilistic reliability index based multidisciplinary design optimization is a nested optimization structure. The outer loop is a deterministic optimization, while the inner loop is a solving process of uncertainty propagation analysis and non-probabilistic reliability index. Considering that the non-gradient regions exist for this reliability index, the global optimization algorithms are always the first choice for solving the NRBMDO problem, which will indeed lead to enormous computation burden. Therefore, in this paper, the decoupled single loop strategy is developed to improve the efficiency.

\subsection{Single loop strategy for NRBMDO (SLS_NRBMDO)}

As stated in refs. [20,21], in order to improve the efficiency, the single loop strategy decouples the nested RBDO with a serial of cycles of deterministic optimization and reliability assessment. The reliability analysis is only conducted after the deterministic optimization. More importantly, this approach adopts the shift strategy to enable all the boundaries of violates constraints to the feasible direction based on the information obtained in the previous cycle.

In this paper, based on the decoupling thought, a single loop strategy for NRBMDO (SLS_NRBMDO) is further developed, which sequentially conducts MDO, UMDA, NRA and translating distance calculation (TDC). To ensure the successful implementation of the scheme, the following key issues need to be tackled with.

(1) The existing MDO strategies, i.e. multidisciplinary feasible method (MDF), concurrent subspace optimization (CSSO), collaborated optimization (CO), bi-level integrated system synthesis (BLISS) and so on, are supposed to deal with the deterministic MDO problem. 


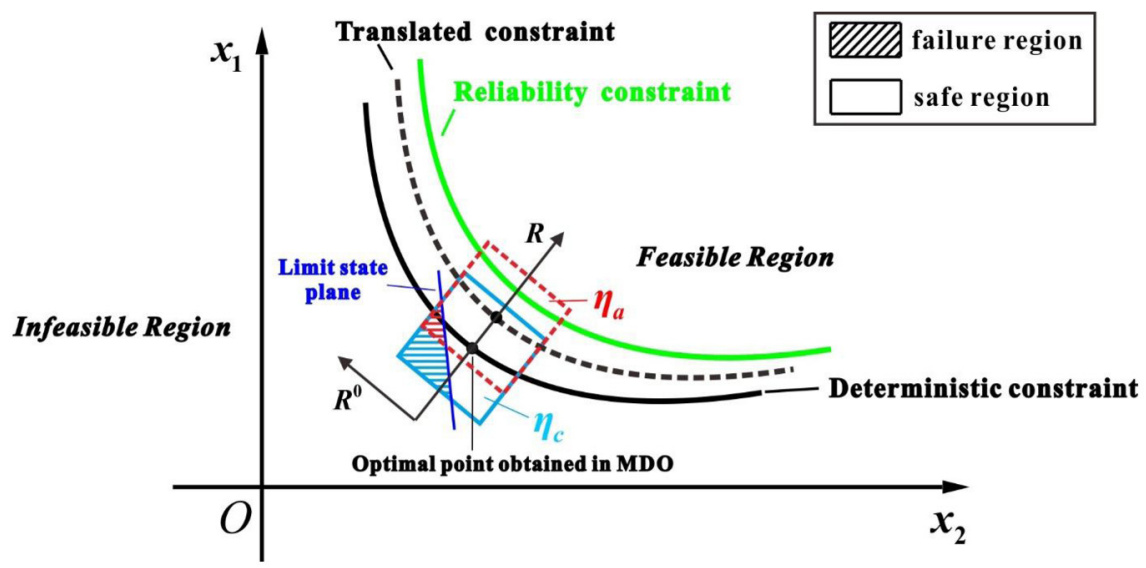

Fig. 5. The sketch of translating limit-state plane in the proposed approach.

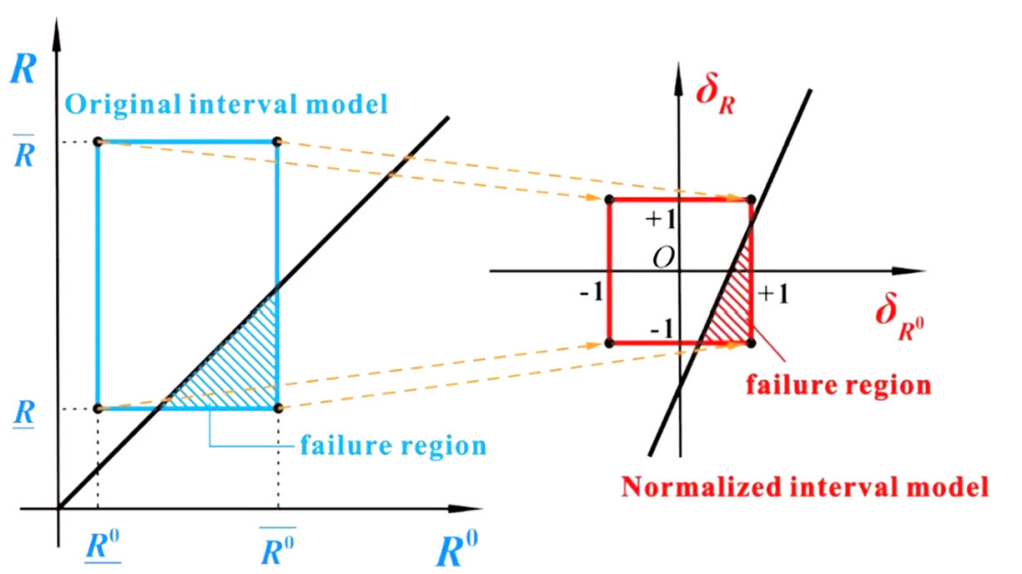

Fig. 6. The relationship between the original interval model and the normalized interval model.

(2) In order to efficiently assess the reliability of multidisciplinary system, the uncertainty propagation analysis in multidisciplinary system is the key issue to be solved. In the paper, as stated in section 3.1, three uncertainty propagation analysis methods, i.e. interval Taylor expansion method, interval vertex method and the optimization method are developed to guarantee the computational accuracy and efficiency.

(3) As claimed in section 3.2, a novel non-probabilistic reliability index based on the volume ratio theory and the expanded non-probabilistic set-theoretic stress-strength interference model is introduced to evaluate the non-probabilistic reliability.

(4) The most important part in the proposed strategy is translating the boundaries of violated constraints based on the reliability information acquired in previous circle. In the following section, the expressions of the translating distance are analytically deduced.

\subsection{Derivation of the translating distance based on performance measurement approach}

In the loop of the deterministic MDO for SLS_NRBMDO, some of the constraints may not satisfy the specified reliability requirements. Under this circumstances, inspired by Ref. [21], we translate the constraints without shape change to exactly meet the target reliability, and then, the non-probabilistic reliability increases from the current reliability $\eta_{c}$ to the allowable reliability $\eta_{a}$. Fig. 5 illustrates the translation strategy when two design variables involved. This approach allows a quick conversion of a reliability constraint to an equivalent approximate deterministic constraint, thus signif- icantly reduces the computation amount caused by an inner loop of UMDA and NRA.

In order to make the analytic process more clearly, the interval model is converted into the normalized space. The relationship between the original interval model and the normalized interval model can be shown in Fig. 6 and mathematically described as follows:

$\left\{\begin{array}{l}\delta_{R}=\left(R-R^{c}\right) / R^{r}, \quad \text { where } R^{c}=(\bar{R}+\underline{R}) / 2 \\ \quad \text { and } R^{r}=(\bar{R}-\underline{R}) / 2 \\ \delta_{R^{0}}=\left(R^{0}-R^{0 c}\right) / R^{0 r}, \text { where } R^{0 c}=\left(\overline{R^{0}}+\underline{R^{0}}\right) / 2 \\ \quad \text { and } R^{r}=\left(\overline{R^{0}}-\underline{R^{0}}\right) / 2\end{array}\right.$

where $\delta_{R}$ and $\delta_{R^{0}}$ are the normalized variables; $R^{c}$ and $R^{0 c}$ are the center values of $R$ and $R^{0} ; R^{r}$ and $R^{0 r}$ are the radius of $R$ and $R^{0}$. Obviously, just as displayed in Fig. $6, \delta_{R} \in[-1,+1]$ and $\delta_{R^{0}} \in[-1,+1]$.

Hence, the initial limit-state function, namely, the performance function can be rewritten as follows by substituting Eq. (31) into Eq. (27).

$G\left(\delta_{R}, \delta_{R^{0}}\right)=R^{r} \delta_{R}-R^{0 r} \delta_{R^{0}}+\left(R^{c}-R^{0 c}\right)$

And, the translated limit-state function with distance $d$ can be formulated as

$G\left(\delta_{R}, \delta_{R^{0}}\right)=R^{r} \delta_{R}-R^{0 r} \delta_{R^{0}}+\left(R^{c}-R^{0 c}\right)+d$

Then, we can deduce the translational distance by borrowing the basic idea of performance measure approach (PMA) [45]. In 


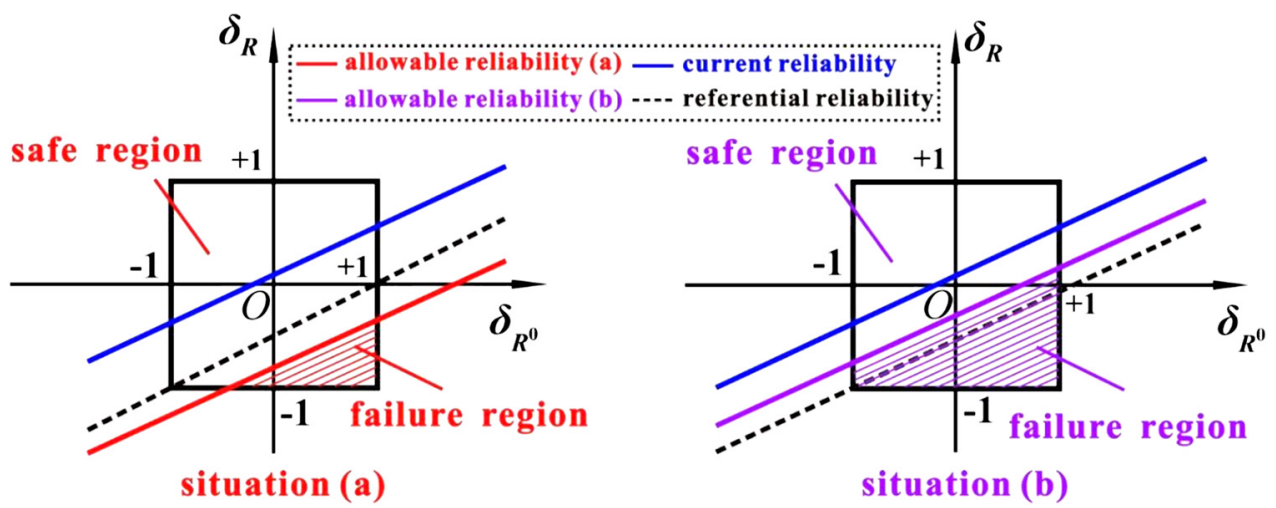

Fig. 7. The sketch of translating constrain condition when $R^{0 r} / R^{r}>1$.

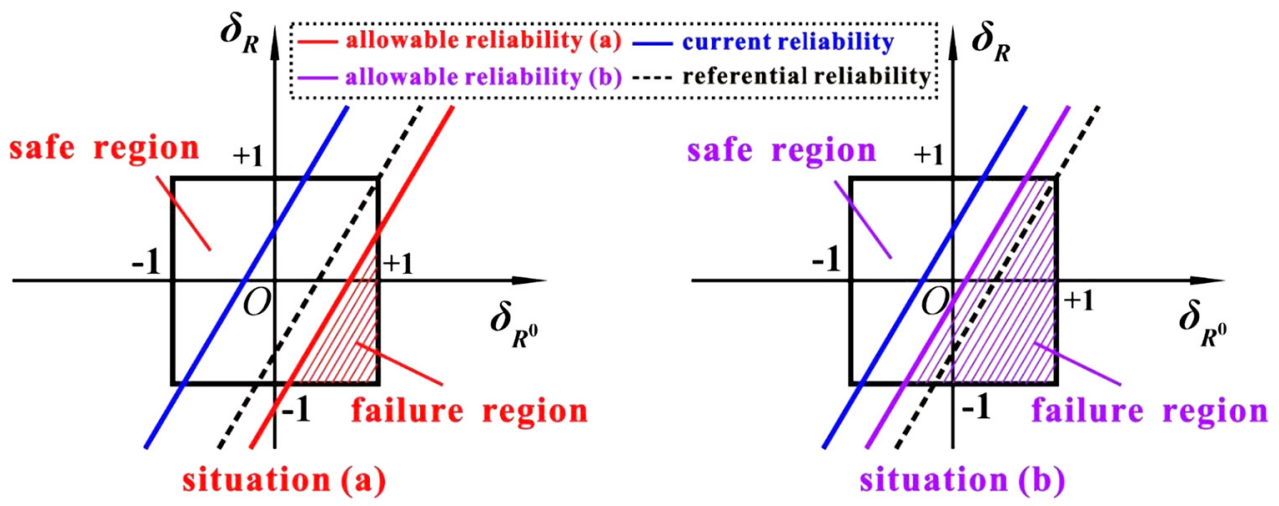

Fig. 8. The sketch of translating constrain condition when $R^{0 r} / R^{r} \leq 1$.

contrast to the reliability index approach (RIA) relying on evaluations of the nonprobabilistic reliability index of the current design, the PMA determines whether a reliability constraint is satisfied according to the sign of the performance. It is found that the PMA is more efficient and stable than the regular RIA according to Ref. [45]. Therefore, the PIM is utilized to tackle this issue.

On this occasion, the translated limit-state function is the performance function corresponding to the allowable reliability $\eta_{a}$. And then, the expressions the translational distance can be derived according to the different interference situations as follows.

\section{(1) Condition I: $R^{0 r} / R^{r}>1$}

As shown in Fig. 7, when $R^{0 r} / R^{r}>1$, the failure region is constructed with either right-angled triangle or right-angled trapezoid. Considering that, two situations need to be accounted according to the states of allowable reliability, corresponding to the situation (a) and (b) in Fig. 7. In order to express the problem clearly and conveniently, the referential reliability $\eta_{r}$, which means that the limit-state plane goes through the point $(-1,-1)$, is introduced. Apparently, $\eta_{r}$ can be calculated as

$\eta_{r}=1-R^{r} /\left(2 R^{0 r}\right)$

In situation (a), the allowable reliability is no less than the referential reliability, namely, $\eta_{a} \geq \eta_{r}$. Then, corresponding to the translated limit-state plane, the failure region constructed of a right-angled triangle can be calculated as:

$S_{\text {failure }}=\frac{\left(R^{r}+R^{0 r}-R^{c}+R^{0 c}-d\right)^{2}}{2 R^{r} R^{0 r}}$

Thus, the allowable non-probabilistic reliability can be formulated as
$\eta_{a}=1-\frac{S_{\text {failure }}}{S_{\text {total }}}=1-\frac{\left(R^{r}+R^{0 r}-R^{c}+R^{0 c}-d\right)^{2}}{2 R^{r} R^{0 r}} / 4$

Then, the distance $d$ can be obtained by solving Eq. (38) as follows:

$d=R^{r}+R^{0 r}-R^{c}+R^{0 c}-\sqrt{8 R^{0 r} R^{r}\left(1-\eta_{a}\right)}$

Besides, in situation (b), the allowable reliability is less than the referential reliability, namely, $\eta_{a}<\eta_{r}$. Then, the failure region constructed of a right-angled trapezoid can be calculated as:

$S_{\text {failure }}=\frac{2\left(R^{0 r}+R^{0 c}-R^{c}-d\right)}{R^{0 r}}$

Thus, the allowable non-probabilistic reliability can be formulated as

$\eta_{a}=1-\frac{S_{\text {failure }}}{S_{\text {total }}}=1-\frac{2\left(R^{0 r}+R^{0 c}-R^{c}-d\right)}{R^{0 r}} / 4$

Eventually, the distance $d$ can be obtained by solving Eq. (41) as follows:

$d=\left(2 \eta_{a}-1\right) R^{0 r}+R^{0 c}-R^{c}$

(2) Condition II: $R^{0 r} / R^{r} \leq 1$

As shown in Fig. 8, when $R^{0 r} / R^{r} \leq 1$, similarly with condition I, two situations need to be considered according to the states of allowable reliability $\eta_{a}$. Under this circumstances, the referential reliability can be also easily obtained, $\eta_{r}=1-R^{0 r} /\left(2 R^{r}\right)$.

In situation (a), the allowable reliability is no less than the referential reliability, namely, $\eta_{a} \geq \eta_{r}$. The failure region is constructed of a right-angled triangle and the allowable non-probabilistic reliability can be calculated as 


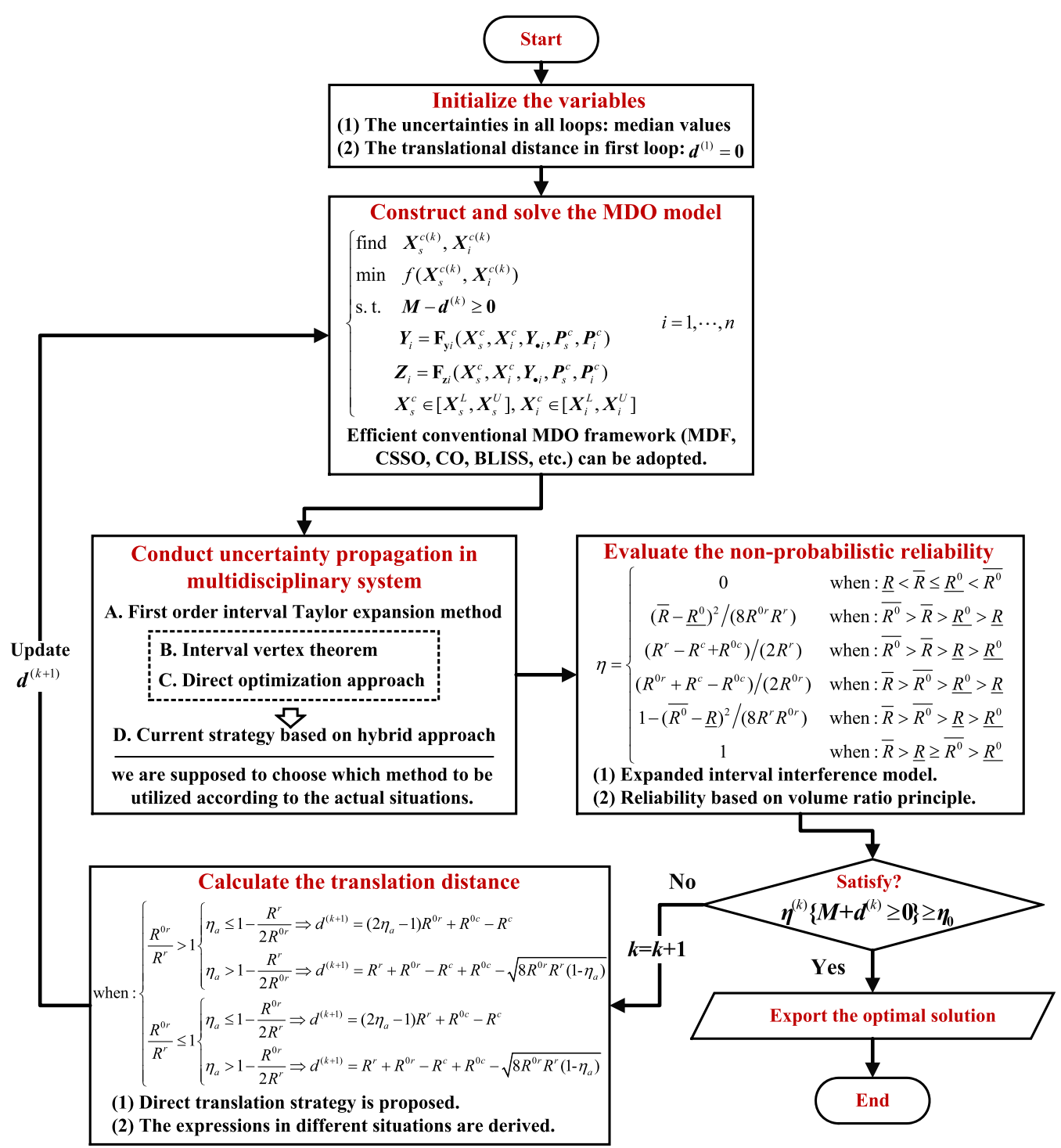

Fig. 9. Flowchart of SLS_NRBMDO.

$\eta_{a}=1-\frac{S_{\text {failure }}}{S_{\text {total }}}=1-\frac{\left(R^{r}+R^{0 r}-R^{c}+R^{0 c}-d\right)^{2}}{2 S^{r} R^{r}} / 4$

Then, the distance $d$ can be obtained by solving Eq. (43) as follows:

$d=R^{r}+R^{0 r}-R^{c}+R^{0 c}-\sqrt{8 R^{0 r} R^{r}\left(1-\eta_{a}\right)}$

Similarly, in situation (b), the allowable reliability is less than the referential reliability, namely, $\eta_{a}<\eta_{r}$. Then, the failure region is constructed of a right-angled trapezoid and the allowable nonprobabilistic reliability can be calculated as

$\eta_{a}=1-\frac{S_{\text {failure }}}{S_{\text {total }}}=1-\frac{2\left(R^{r}+R^{0 c}-R^{c}-d\right)}{R^{r}} / 4$

Eventually, the distance $d$ can be obtained by solving Eq. (45) as follows:

$d=\left(2 \eta_{a}-1\right) R^{r}+R^{0 c}-R^{c}$

All in all, the mathematical expressions of the translating distance can be summarized as

$$
\text { when: }\left\{\begin{array}{c}
\frac{R^{0 r}}{R^{r}}>1\left\{\begin{array}{l}
\eta_{a} \leq 1-\frac{R^{r}}{2 R^{0 r}} \Rightarrow \\
d=\left(2 \eta_{a}-1\right) R^{0 r}+R^{0 c}-R^{c} \\
\eta_{a}>1-\frac{R^{r}}{2 R^{0 r}} \Rightarrow d=R^{r}+R^{0 r}-R^{c}+R^{0 c} \\
-\sqrt{8 R^{0 r} R^{r}\left(1-\eta_{a}\right)}
\end{array}\right. \\
\frac{R^{0 r}}{R^{r}} \leq 1\left\{\begin{array}{l}
\eta_{a} \leq 1-\frac{R^{0 r}}{2 R^{r}} \Rightarrow \\
d=\left(2 \eta_{a}-1\right) R^{r}+R^{0 c}-R^{c} \\
\eta_{a}>1-\frac{R^{0 r}}{2 R^{r}} \Rightarrow d=R^{r} \\
-\sqrt{8 R^{0 r} R^{r}\left(1-\eta_{a}\right)}
\end{array}\right.
\end{array}\right.
$$

\subsection{Procedures and flowchart of the proposed single loop approach}

In this section, the flowchart of the proposed approach is illustrated in Fig. 9 and the procedure is elaborated in detail as follows:

Step 1: Initialize the variables. In the proposed strategy, the values of the uncertainties are set to be the median values. Moreover, in view of that there is no information about the nonprobabilistic reliability and the translating distance for the deter- 
ministic optimization in the first loop, the shift distance in the first loop is set to be $\boldsymbol{d}^{(1)}=\mathbf{0}$.

Step 2: Construct and solve the MDO model. Translate the constraints in the direction of feasible region with distance $\boldsymbol{d}^{(k)}$. Construct the deterministic MDO problem and the optimization model can be mathematically written as Eq. (48) shows when the strategy of MDF is applied. The initial value of $k$ is supposed to be 1 .

$$
\begin{cases}\text { find } & \boldsymbol{X}_{s}^{c(k)}, \boldsymbol{X}_{i}^{c(k)} \\ \min & f\left(\boldsymbol{X}_{s}^{c(k)}, \boldsymbol{X}_{i}^{c(k)}\right) \\ \text { s.t. } & \boldsymbol{M}-\boldsymbol{d}^{(k)} \geq \mathbf{0} \\ & \boldsymbol{Y}_{i}=\mathbf{F}_{\mathbf{y} i}\left(\boldsymbol{X}_{s}^{c}, \boldsymbol{X}_{i}^{c}, \boldsymbol{Y}_{\bullet i}, \boldsymbol{P}_{s}^{c}, \boldsymbol{P}_{i}^{c}\right) \\ & \boldsymbol{Z}_{i}=\mathbf{F}_{\mathbf{z i}}\left(\boldsymbol{X}_{s}^{c}, \boldsymbol{X}_{i}^{c}, \boldsymbol{Y}_{\bullet i}, \boldsymbol{P}_{s}^{c}, \boldsymbol{P}_{i}^{c}\right) \\ & \boldsymbol{X}_{s}^{c} \in\left[\boldsymbol{X}_{s}^{L}, \boldsymbol{X}_{s}^{U}\right], \boldsymbol{X}_{i}^{c} \in\left[\boldsymbol{X}_{i}^{L}, \boldsymbol{X}_{i}^{U}\right]\end{cases}
$$

Note that, in the $k$-th loop, the optimization is the regular MDO with the median values of all the uncertain parameters. The deterministic MDO problem can be solved through conventional MDO framework, such as MDF, CSSO, CO, BLISS, etc. Consequentially, the optimal point $\left(\boldsymbol{X}_{s}^{c(k)}, \boldsymbol{X}_{i}^{c(k)}\right)$ can be obtained.

Step 3: Conduct uncertainty propagation in multidisciplinary system. Based on the optimal point obtained in $k$-th loop, that is, $\left(\boldsymbol{X}_{s}^{c(k)}, \boldsymbol{X}_{i}^{c(k)}\right)$, the interval range of the outputs $\boldsymbol{Z}_{i}^{(k)}$ can be acquired through the uncertainty analysis methods proposed in section 3.1.

Step 4: Evaluate the non-probabilistic reliability. Based on the expanded non-probabilistic set-theoretic stress-strength interference model and volume ratio principle, the reliability can be easily calculated through Eq. (30) derived in section 3.2. Then the reliability vector $\eta_{i}^{(k)}$ of all constraint conditions is acquired.

Step 5: Check the convergence. If all elements of the obtained reliability vector $\boldsymbol{\eta}_{i}$ meet the reliability requirements, the entire optimization process stops and the optimal point $\left(\boldsymbol{X}_{s}^{c(k)}, \boldsymbol{X}_{i}^{c(k)}\right)$ is perceived as the final optimal solution; otherwise, turn to step 5 .

Step 6: Calculate the translating distances of the constraints. As some of the constraints may not satisfy specified reliability requirements, we translate the constraints in the direction of feasible region. According to the different situations of the constraints, the translation distances $\boldsymbol{d}^{(k)}$ can be calculated by utilizing the strategies proposed in section 4.2 .

Step 7: Loop. Conduct step 2 to step 6 until the convergence is reached.

All in all, by using SLS_NRBMDO, the nested optimization is avoided and the convergence problem in traditional NRBMDO caused by the non-gradient region of reliability is solved, for that the non-probabilistic reliability no longer act as constraints in optimization model. Therefore, the two problems stated in section 1 can be well solved by the proposed decoupling strategy.

\section{Demonstrative examples}

In this section, the proposed SLS_NRBMDO is applied in both numerical example and engineering example. The regular MDO and UMDO methods are also utilized in the numerical example to illustrate the effectiveness and high efficiency of the proposed decoupled approach.

\subsection{Case I: numerical example}

The multidisciplinary system consisting of two subsystems, which are discipline1 and discipline2, is considered in this case. $Z, X_{1}, X_{2}$ are design variables. $x_{s}, x_{1}, x_{2}$ are uncertain variables. The allowable reliability vector is set to be $\eta_{0}=(0.9,0.9)$. The functional relationships as well as the uncertain descriptions are displayed in Eq. (47):

$$
\begin{aligned}
& \text { find } Z, X_{1}, X_{2} \\
& \text { minimize } F=f_{1}+f_{2} \\
& \text { subject to: } \\
& \text { discipline 1: } 4 y_{12}=2 Z+2 p_{s}-3 X_{1}+2 x_{2}-4 y_{21} \\
& \text { objective 1: } f_{1}=0.5\left(Z+\bar{x}_{s}\right)^{2}+X_{1}^{2} \\
& \text { constraint 1: }\left\{\begin{array}{c}
g_{1}=Z+p_{s}+2 X_{1}+2 y_{21}-p_{1} \\
\eta_{1}\left(g_{1}-g_{1}^{0} \geq 0\right) \geq 0.9
\end{array}\right. \\
& \text { discipline 2: } 4 y_{21}=-2 Z-2 p_{s}-X_{1}+2 X_{2}+4 y_{12} \\
& \text { objective } 2: f_{2}=0.5\left(Z+\bar{x}_{s}\right)^{2}+X_{2}^{2} \\
& \text { constraint 2: }\left\{\begin{array}{c}
g_{2}=5 Z-5 p_{s}+3 X_{2}-4 y_{21}-p_{2} \\
\eta_{2}\left(g_{2}-g_{2}^{0} \geq 0\right) \geq 0.9
\end{array}\right. \\
& \text { design variables: } \begin{array}{l}
0 \leq X_{1}, X_{2} \leq 5 \\
1 \leq Z \leq 5
\end{array} \\
& p_{s} \in[0.90,1.10] \text {; } \\
& p_{1} \in[4.05,4.95] \text {; } \\
& \text { uncertain parameters: } p_{2} \in[0.95,1.05] \\
& g_{1}^{0} \in[-0.60,0] \\
& g_{2}^{0} \in[-1.00,1.00]
\end{aligned}
$$

\subsubsection{Optimization design based regular MDO}

In the determine optimization, the uncertain effect is neglected and all the uncertainties are set as the median values. Thus, based on Eq. (49), the coupled multidisciplinary analysis model and optimization model can be shown in Eq. (48) and Eq. (49) respectively.

$$
\begin{aligned}
& \begin{cases}4 y_{12}=2 Z+2 x_{s}-3 X_{1}+2 X_{2}-4 y_{21} \\
4 y_{21}=-2 Z-2 x_{s}-X_{1}+2 X_{2}+4 y_{12}\end{cases} \\
& \begin{cases}\min & F=f_{1}+f_{2}=(Z+1)^{2}+X_{1}^{2}+X_{2}^{2} \\
\text { s.t. } & g_{1}=\left(Z+1+2 X_{1}+2 y_{21}\right)-4.5 \geq 0 \\
& g_{2}=\left(5 Z-5+3 X_{2}-4 y_{12}\right)-1 \geq 0\end{cases} \\
& \quad 0 \leq Z \leq 51 \leq X_{1}, X_{2} \leq 5
\end{aligned}
$$

In the problem, MDF is utilized to solve the optimization design problem. The converging process can be shown by the blue line in Fig. 10. The obtained optimal point is $\left(X_{1}, X_{2}, Z\right)=$ $(1.2500,1.6261,0.6239)$ and the minimum value of objective function is $F=6.8438$. Note that the non-probabilistic reliability at the optimal point can be calculated based on the volume ratio theory when the uncertainties are considered and the reliability vector is $\boldsymbol{\eta}=\left(\eta_{1}, \eta_{2}\right)=(0.7708,0.8125)$. Obviously, the reliability requirements are not satisfied.

\subsubsection{Optimization based on Regular UMDO}

Based on Eq. (49), uncertain multidisciplinary design feasible method (UMDF) is utilized to solve the optimization design problem. The converging process can be shown in black line in Fig. 10. The obtained optimal point is $\left(X_{1}, X_{2}, Z\right)=$ $(1.4289,1.6287,0.6292)$ and the minimum value of objective function is $F=7.3483$. Note that the reliability vector at the optimal point can be calculated as $\eta=\left(\eta_{1}, \eta_{2}\right)=(0.9,0.9)$, which means that the reliability requirements have already been satisfied. 


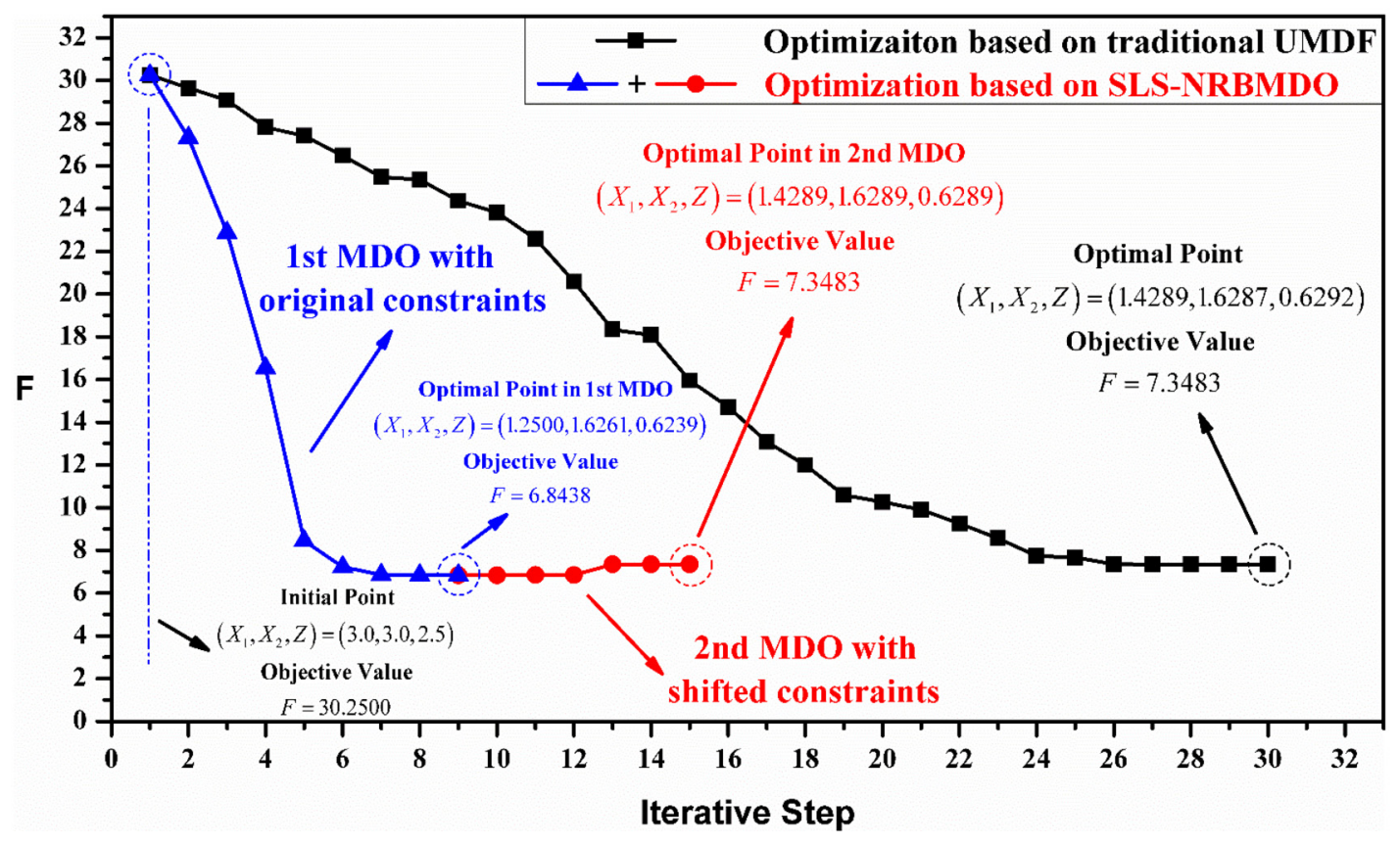

Fig. 10. The converging process of traditional UMDF and SLS_NRBMDO.

Table 1

The optimum solutions of SLS_NRBMDO and traditional UMDF.

\begin{tabular}{|c|c|c|c|c|c|c|c|}
\hline & \multicolumn{3}{|c|}{ Design variables } & \multirow{2}{*}{$\frac{\text { Object }}{F}$} & \multicolumn{2}{|c|}{ Constrain reliability } & \multirow{2}{*}{$\begin{array}{l}\text { Total } \\
\text { multidisciplinary } \\
\text { analyses }\end{array}$} \\
\hline & $X_{1}$ & $X_{2}$ & $Z$ & & $\eta_{1}$ & $\eta_{2}$ & \\
\hline SLS_NRBMDO & 1.4289 & 1.6287 & 0.6292 & 7.3483 & 0.9 & 0.9 & 47 \\
\hline UMDF & 1.4289 & 1.6289 & 0.6289 & 7.3483 & 0.9 & 0.9 & 388 \\
\hline
\end{tabular}

\subsubsection{Optimization based on the proposed approach}

In this section, the proposed sing loop strategy is applied to solve this optimization problem and the analysis procedure can be organized as follows:

(1) Initial the values of design variables, coupled variables and reliability index;

(2) In the first circle, the deterministic optimization model can be formulated as Eq. (50) and Eq. (51). The optimal solution can be easily obtained as $X_{1}=1.2500, X_{2}=1.6261$ and $Z=0.6239$ through MDF.

(3) Based on stress-strength interference model, the reliability of the constraints can be calculated as $\boldsymbol{\eta}=\left(\eta_{1}, \eta_{2}\right)=$ $(0.7708,0.8125)$, both of which are lower than the required values, which are, $\eta_{0}=(0.9,0.9)$.

(4) Translate the limit-state plane with distance $\boldsymbol{d}=\left(d_{1}, d_{2}\right)=$ $(0.1867,0.2023)$, which is calculated based on the reliability information obtained in step (3) and theory proposed in section 4.2 .

(5) Reformulate the deterministic optimization problem for the second circle. Substitute $\boldsymbol{d}$ into Eq. (48), and the optimal solution can be easily obtained as $X_{1}=1.4289, X_{2}=1.6287$ and $Z=0.6292$ through MDF. In the optimization problem, the initial value of design variables is set to be the optimal point obtained in first circle to accelerate the convergence.

(6) The reliability of the constraints can be calculated as $\boldsymbol{\eta}=$ $\left(\eta_{1}, \eta_{2}\right)=(0.9,0.9)$, which means that the reliability requirements are satisfied and the convergence is achieved. The converging process can be shown in red line in Fig. 10. Then, the optimal point obtained in the second circle is considered to be the optimal solutions to this RBMDO problem, and the minimum value of objective function is $F=7.3483$.
The solutions of SLS_NRBMDO are compared with regular UMDF, which nested the uncertainty analysis into deterministic optimization. Obviously, both of the two methods can obtain the optimal solution. However, as listed in Table 1, the function calls of the nested method are significantly more than those of SLS_NRBMDO, which indeed verify the efficiency of SLS_NRBMDO. And the comparison of the converging processes between the traditional UMDO and SLS_NRBMDO can be clear expounded in Fig. 10.

\subsection{Case II: design of hypersonic wing}

In this case, the reliability based dimensional design of the hypersonic wing is chosen as the practical engineering application to demonstrate the efficiency of SLS_NRBMDO. Considering that the mechanical state of hypersonic wing in harsh multi-field coupled environment during the re-entry process is one of the major concerns for the high flight speed. Therefore, in this case, the hypersonic wing is modeled as a multidisciplinary system where fluid and structures are highly coupled as shown in Fig. 11 to obtain the accurate responses. Obviously, there are two design variables $\left(L_{r i b}, L_{\text {beam }}\right)$, two linking variables (Aerodynamic Shape, Aerodynamic Pressure), two system outputs $\left(d_{\max }^{\text {actual }}, \sigma_{\max }^{a c t u a l}\right)$ in the coupled fluidstructure system.

In addition, as shown in Fig. 12, three components, namely, skin, frame and honeycomb sandwiches, constitute the typical hypersonic wing structure. Specifically, (1) the wing surface is covered by titanium alloy skin so as to maintain the aerodynamic configuration; (2) the frame structure is made up of titanium alloy ribs and beams. (3) The sandwiches made with honeycomb paperboard are filled between ribs and beams. All the three components are connected together by rivet joints and glue. And for the bound- 


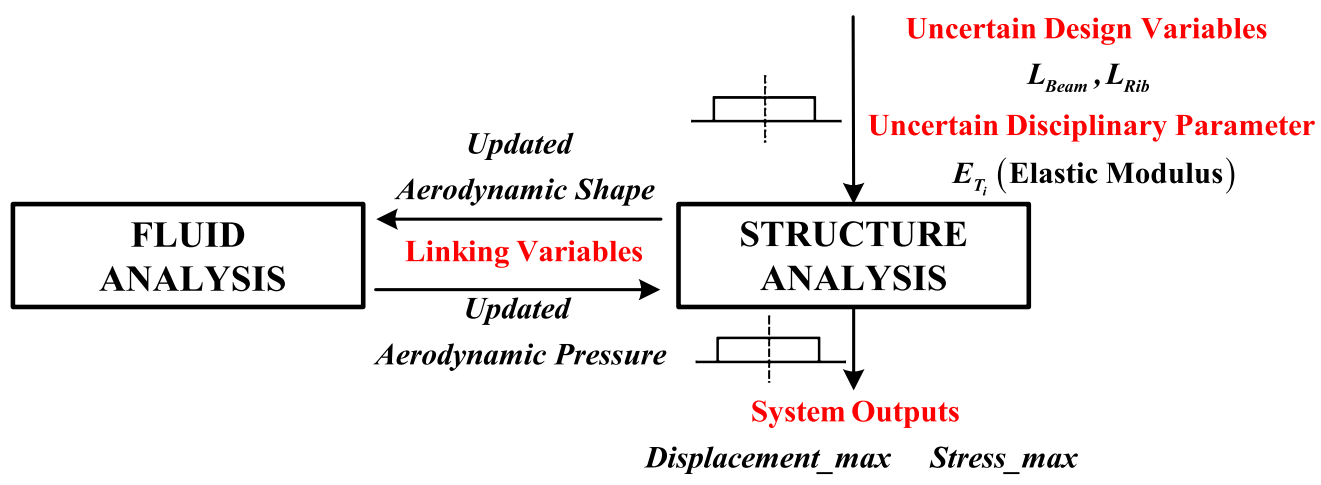

Fig. 11. Information flow diagram of the engineering case.

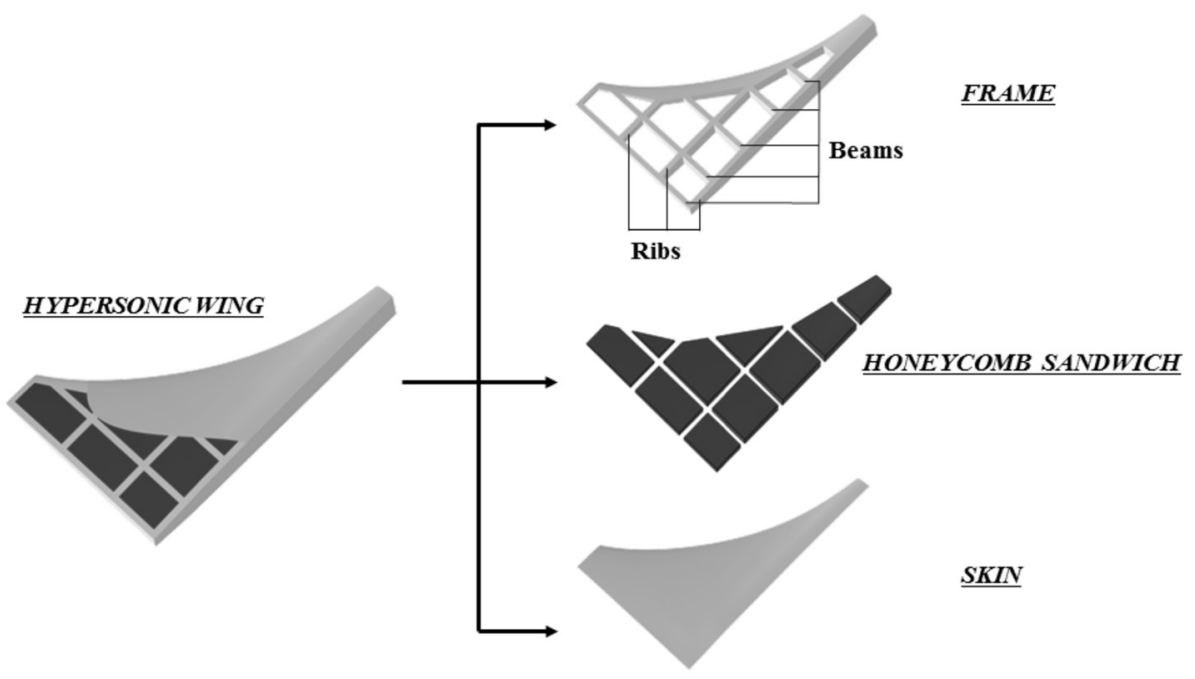

Fig. 12. Illustration of the components for the hypersonic wing.

Table 2

Uncertain descriptions of input variables in case II.

\begin{tabular}{llllll}
\hline & $L_{\text {rib }}$ & $L_{\text {beam }}$ & $E_{T i}$ & $d_{\max }^{\text {allowable }}$ & $\sigma_{\max }^{\text {allowable }}$ \\
\hline Median value & $20 \mathrm{~mm} \sim 50 \mathrm{~mm}$ & $20 \mathrm{~mm} \sim 50 \mathrm{~mm}$ & $1.0 \mathrm{e}-11 \mathrm{~Pa}$ & $20 \mathrm{~mm}$ & $1.0 \mathrm{e}-9 \mathrm{~Pa}$ \\
Radius & $0.4 \mathrm{~mm}$ & $0.4 \mathrm{~mm}$ & $5.0 \mathrm{e}-10 \mathrm{~Pa}$ & $0.4 \mathrm{~mm}$ & $5 \mathrm{e}-7 \mathrm{~Pa}$ \\
\hline
\end{tabular}

ary conditions, the region which connects to fuselage is imposed by fixed constraints.

Moreover, in view of material dispersion, machining error, uncertainty in measurement, cognitive bias etc. in practical engineering, we assume that elastic modulus of alloy materials $\left(E_{T i}\right)$, the thickness of the ribs $\left(L_{\text {rib }}\right)$ and beams $\left(L_{\text {beam }}\right)$, the allowable displacement $\left(d_{\max }^{\text {allowable }}\right)$ and the allowable stress $\left(\sigma_{\max }^{\text {allowable }}\right)$ are uncertain variables which are specifically described in Table 2.

In this case, $\eta_{\text {dis }}$ denotes the non-probabilistic reliability that the actual maximum displacement $d_{\max }^{\text {actual }}$ is less than the allowable displacement $d_{\max }^{\text {allowable }} \eta_{\text {stress }}$ denotes the non-probabilistic reliability that the actual maximum stress $\sigma_{\max }^{\text {actual }}$ is less than the allowable displacement $\sigma_{\max }^{\text {allowable }}$. The above reliability constraints are used to guide the design of the hypersonic wing and the expression of the reliability can be explained as

$$
\begin{aligned}
& \eta_{\text {dis }}=\eta\left(d_{\max }^{\text {allowable }}-d_{\max }^{\text {actual }} \geq 0\right) \\
& \eta_{\text {stress }}=\eta\left(\sigma_{\max }^{\text {allowable }}-\sigma_{\max }^{\text {actual }} \geq 0\right)
\end{aligned}
$$

The procedure of reliability-based design for hypersonic wing through SLS_NRBMDO is represented in Fig. 13. In order to settle this complex engineering problem and implement the proce- dure successfully, the solution strategy can be carried out by the integration of CATIA which provides parametric geometry modeling technology, ICEM which offers parametric CFD modeling technology, ANSYS which conducts finite element analysis, MATLAB which calculates the non-probabilistic reliability and the translating distance, the self-compiled program which calculates the aerodynamic pressure on the skin surface. All the module interface strategies for data exchange can be achieved by iSIGHT as displayed in Fig. 14.

It should be pointed out that the flight environment in this case can be described as: (1) the altitude is $30 \mathrm{~km}$; (2) the free stream Mach number is 6; (3) the angle of attack is $16^{\circ}$. Besides, considering that the system outputs, namely, $d_{\max }^{\text {actual }}$ and $\sigma_{\max }^{\text {actual }}$ are monotonous to the input variables, namely, $L_{r i b}$ and $L_{\text {beam }}$, in the aerodynamic and structural coupling analysis. Hence, the interval vertex theorem is supposed to be applied in this case and the theorem is accomplished by utilizing the design of experiment (DOE) tool to obtain the interval vertex array.

Note that, in this complex engineering problem, the traditional UMDO methods is no longer suitable due to the huge amount of computation and the convergence difficulty. In this case, by utilizing SLS_NRBMDO, both the accuracy and efficiency of the NRBMDO 


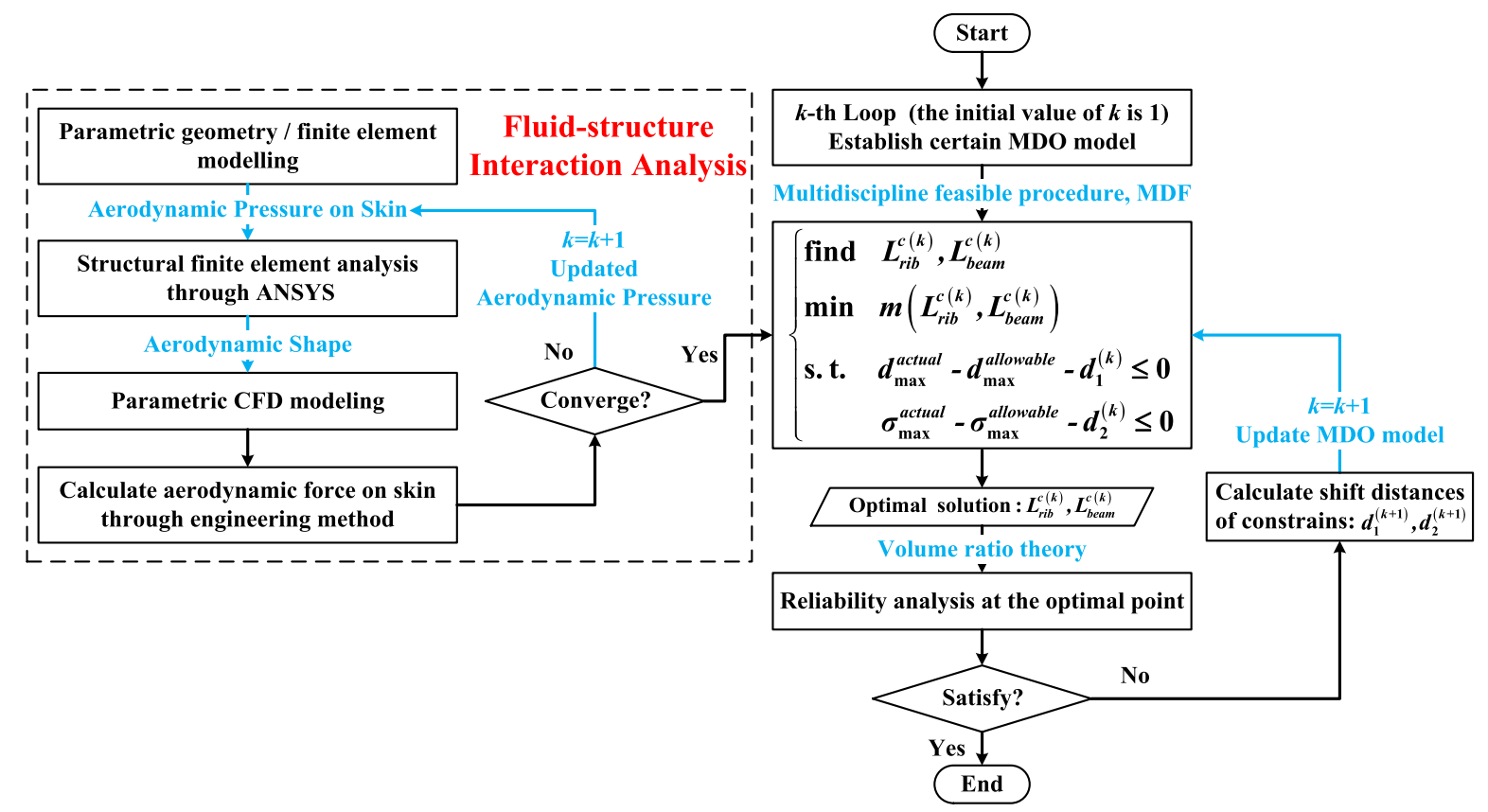

Fig. 13. Procedure of reliability-based design for hypersonic wing through SLS_NRBMDO.

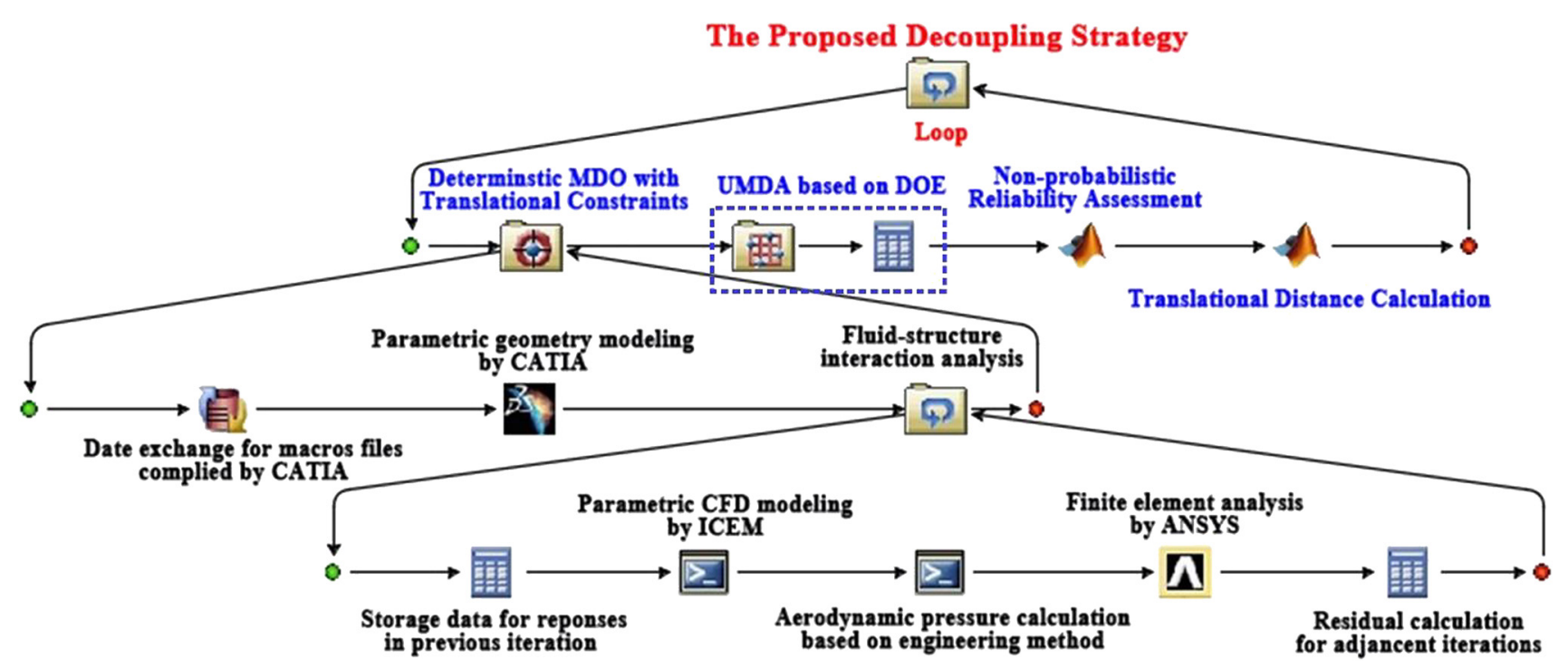

Fig. 14. Illustration of the integration platform for SLS_NRBMDO based on iSIGHT.

Table 3

Iterative process of SLS_NRBMDO.

\begin{tabular}{|c|c|c|c|c|c|c|c|c|c|}
\hline Step & $L_{\text {beam }}$ & $L_{\text {rib }}$ & $m$ & $\eta_{\text {dis }}$ & $\eta_{\text {stress }}$ & $d_{\max }^{c}$ & $\sigma_{\max }^{c}$ & $d_{1}$ & $d_{2}$ \\
\hline 1 & 0.02605 & 0.02000 & 590.2454 & 0.4999 & 0.7067 & 0.02000 & $9.7933 e-8$ & $1.7590 \mathrm{e}-3$ & $3.0096 e-7$ \\
\hline 2 & 0.03618 & 0.02000 & 630.3865 & 0.9843 & 0.9993 & 0.02000 & $9.7433 e-8$ & $7.5943 e-5$ & $-3.9516 e-6$ \\
\hline 3 & 0.03661 & 0.0200 & 632.0676 & 0.9898 & 1.0000 & 0.02000 & $9.6886 e-8$ & $3.2938 \mathrm{e}-6$ & $-5.4194 \mathrm{e}-6$ \\
\hline 4 & 0.03661 & 0.0201 & 632.1715 & 0.9900 & 1.0000 & 0.02000 & $9.6337 e-8$ & & \\
\hline
\end{tabular}

problem can be ensured. The analysis results are listed in Table 3 . Obviously, it needs only 4 steps of MDO, UMDA, and NRA, as well as 3 steps of TDC, then the optimal solution of UMDO can be obtained. Moreover, the convergence process can be illustrated in Fig. 15.

\subsection{Discussion}

From the results of the above two cases, the paper comes to the following conclusions:
(1) As shown in cases I, compared to the traditional double level UMDO strategy, SLS_NRBMDO has a higher efficiency for the reason that the reliability analysis and MDO are decomposed and conducted in a recursive manner. Furthermore, in SLS_NRBMDO, efficient UMDA methods are introduced, rational non-probabilistic reliability index is proposed, and the expression of the translating distance for the deterministic constraint is mathematically deduced. Thus the computational efficiency is further improved for SLS_NRBMDO. 


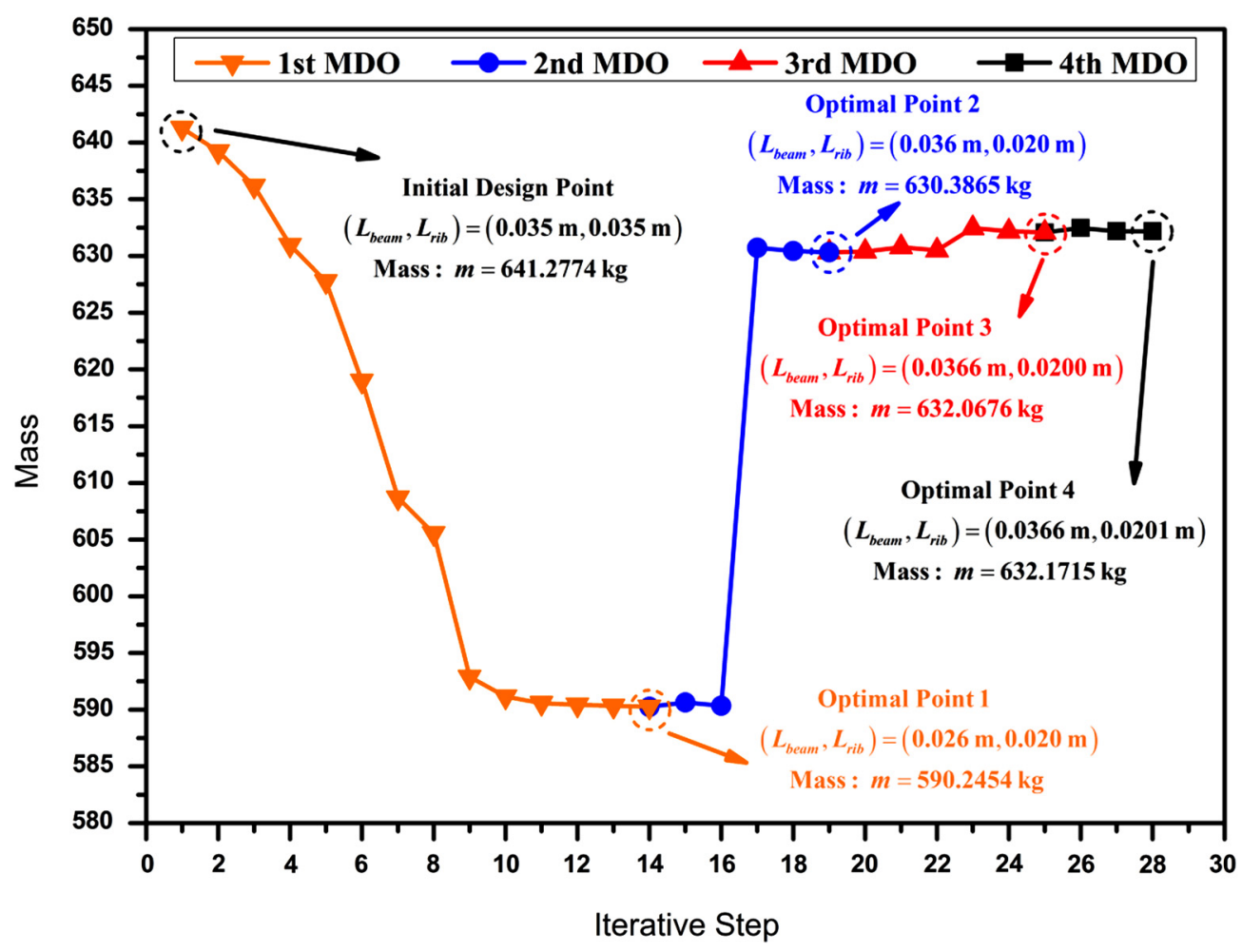

Fig. 15. The converging process of hypersonic wing design through SLS_NRBMDO.

(2) As cases II demonstrated, in actual engineering problems, the traditional UMDO methodology is always not suitable due to the unbearable computational burden. In such a situation, the proposed SLS_NRBMDO can be a good choice. And by utilizing SLS_NRBMDO, both the accuracy and efficiency can be guaranteed.

(3) As both case I and case II indicated, in the proposed strategy of SLS_NRBMDO, the optimal point obtained in the $k$-th MDO is taken as the initial design point the next circle, that is, the $(k+1)$-th MDO. Consequently, the efficient gradient based optimization algorithms can be utilized to accelerate the convergence process.

\section{Conclusions}

The tremendous computation and convergence difficulty in NRBMDO always hamper its application in practical engineering. To address these issues, a single loop strategy for solving the NRBMDO problems has been investigated to improve the efficiency. In the proposed approach, MDO, UMDA, NRA and TDC are conducted sequentially and iteratively by updating the translating distance of constrains until the convergence is reached. In order to guarantee the high efficiency of the proposed SLS_NRBMDO method, the following aspects are researched in this work. First, three methods, i.e. the first order interval Taylor expansion method, the interval vertex theorem and the direct optimization approach, have been proposed to efficiently conduct the uncertainty propagation in multidisciplinary system. Then, in order to evaluate the non-probabilistic reasonably in multidisciplinary system, the expanded non-probabilistic set-theoretic stress-strength interference model and the volume ratio theory are introduced in this study. Furthermore, the translating distance based on the proposed non-probabilistic reliability index and the performance measure approach in SLS_NRBMDO is mathematically derived. Apparently, SLS_NRBMDO is likely to converge in a few cycles, and therefore, the computational of efficiency will be much higher than the regular procedure where reliability analysis is applied directly with optimization. And the high efficiency is testified with one numerical example and one engineering case.

Furthermore, considering the fact that there always exist some other types of non-probabilistic uncertainties in practical engineering projects, such as ellipsoid model, hybrid model and so on, a further interesting issue to be investigated is extending the proposed methodology to other non-probabilistic models for uncertain-but-bounded uncertainties to improve the design optimization efficiency for the complex structural systems.

\section{Conflict of interest statement}

None declared.

\section{Acknowledgements}

The authors would like to thank the major research project (No. MJ-F-2012-04),Defense Industrial Technology Development Program (No. JCKY2013601B001, No. JCKY2016601B001, No. JCKY2013205B002) and National Nature Science Foundation of the P.R. China (No. 11372025, No. 11432002, No. 11572024) for the financial supports.

\section{References}

[1] L. Cavagna, S. Ricci, L. Travaglini, NeoCASS: an integrated tool for structura sizing, aeroelastic analysis and MDO at conceptual design level, Prog. Aerosp. Sci. 47 (8) (2011) 621-635.

[2] Z.G. Wang, W. Huang, L. Yan, Multidisciplinary design optimization approach and its application to aerospace engineering, Chin. Sci. Bull. 59 (36) (2014) 5338-5353.

[3] Y. He, J. Mcphee, Multidisciplinary optimization of multibody systems with application to the design of rail vehicles, Multibody Syst. Dyn. 14 (2) (2005) 111-135.

[4] P. Wang, G. Wu, Multidisciplinary design optimization of vehicle instrument panel based on multi-objective genetic algorithm, Chin. J. Mech. Eng. 26 (2) (2013) 304-312. 
[5] F. Mastroddi, M. Tozzi, E. Mastrella, MDO analyses of wing structures for a complete aeroelastically constrained aircraft, CEAS Aeronaut. J. 3 (1) (2012) 67-77.

[6] T.F. Wunderlich, Multidisciplinary wing optimization of commercial aircraft with consideration of static aeroelasticity, CEAS Aeronaut. J. 6 (3) (2015) 407-427.

[7] J. Ahn, J.H. Kwon, Sequential approach to reliability analysis of multidisciplinary analysis systems, Struct. Multidiscip. Optim. 28 (6) (2004) 397-406.

[8] L. Wang, et al., Time-variant reliability model and its measure index of structures based on a non-probabilistic interval process, Acta Mech. 226 (10) (2015) 3221-3241.

[9] W. Yao, et al., Sequential optimization and mixed uncertainty analysis method for reliability-based optimization, AIAA J. 51 (9) (2013) 2266-2277.

[10] H. Agarwal, J.E. Renaud, J.D. Mack, Decomposition approach for reliability-based multidisciplinary design optimization, in: 44th AIAA/ASME/ASCE/AHS/ASC Structures, Structural Dynamics, and Materials Conference, AIAA, 2000.

[11] D.B. Meng, et al., Reliability-based multidisciplinary design optimization using subset simulation analysis and its application in the hydraulic transmission mechanism design, J. Mech. Des. 137 (5) (2015) 9.

[12] Y.S. Zhang, et al., Reliability-based multidisciplinary design optimization for centrifugal compressor using the fourth moment method, in: J.T. Han, Z.Y. Jiang, S. Jiao (Eds.), Advanced Manufacturing Technology, Pts 1, 2, Trans Tech Publications Ltd, Stafa-Zurich, 2011, pp. 575-581.

[13] P.N. Koch, et al., Statistical approximations for multidisciplinary design optimization: the problem of size, J. Aircr. 36 (1) (1999) 275-286.

[14] P.N. Koch, B. Wujek, O. Golovidov, A multi-stage, parallel implementation of probabilistic design optimization in an MDO framework, in: Proceeding of the Eighth AIAA/USAF/NASA/ISSMO Symposium on Multidisciplinary Analysis and Optimization, 2000.

[15] H. Agarwal, et al., A unilevel method for reliability based design optimization, in: Proceedings of the 45th AIAA/ASME/ASCE/AHS Structures, Structural Dynamics, and Materials Conference, 2004.

[16] X. Chen, T.K. Hasselman, N.D. J, Reliability based structural design optimization for practical applications, in: Proceedings of the 38th AIAA/ASME/ASCE/AHS Structures, Structural Dynamics, and Materials Conference, 1997.

[17] J. Liang, Z.P. Mourelatos, J. Tu, A single-loop method for reliability-based design optimization, Int. J. Prod. Dev. 5 (1) (2008) 419-430.

[18] C. Shi, et al., Adaptive decoupling synchronous control of dissimilar redundant actuation system for large civil aircraft, Aerosp. Sci. Technol. 47 (2015) 114-124.

[19] R. Sues, M. Cesare, An innovative framework for reliability-based MDO, in: The 41st AIAA/ASME/ASCE/AHS/ASC Structures, Structural Dynamics, and Materials Conference and Exhibit, 2000.

[20] X. Du, W. Chen, Sequential optimization and reliability assessment method for efficient probabilistic design, in: Proceedings of the ASME 2002 Design Engineering Technical Conference and Computers and Information in Engineering Conference, 2002.

[21] Y.T. Wu, et al., Safety-factor based approach for probability-based design optimization, in: 19th AIAA Applied Aerodynamics Conference, American Institute of Aeronautics and Astronautics, 2001.

[22] X.P. Du, J. Guo, H. Beeram, Sequential optimization and reliability assessment for multidisciplinary systems design, Struct. Multidiscip. Optim. 35 (2) (2008) $117-130$.

[23] L.S. Li, J.H. Liu, S.H. Liu, An efficient strategy for multidisciplinary reliability design and optimization based on CSSO and PMA in SORA framework, Struct. Multidiscip. Optim. 49 (2) (2014) 239-252.
[24] X.D. Zhang, H.Z. Huang, Sequential optimization and reliability assessment for multidisciplinary design optimization under aleatory and epistemic uncertainties, Struct. Multidiscip. Optim. 40 (1-6) (2010) 165-175.

[25] Y. Ben-Haim, Robust reliability of structures, Adv. Appl. Mech. 33 (08) (1997) $1-41$.

[26] Y. Ben-Haim, I. Elishakoff, Convex Models of Uncertainty in Applied Mechanics, Elsevier, Amsterdam, 1990.

[27] Y. Ben-Haim, I. Elishakoff, Discussion on: a non-probabilistic concept of reliability, Struct. Saf. 17 (3) (1995) 195-199.

[28] S. Guo, Z. Lu, Hybrid probabilistic and non-probabilistic model of structural reliability, J. Mech. Strength 24 (4) (2002) 524-526.

[29] X. Wang, Z. Qiu, I. Elishakoff, Non-probabilistic set-theoretic model for structural safety measure, Acta Mech. 198 (1-2) (2008) 51-64.

[30] Y.J. Luo, Z. Kang, A. Li, Structural reliability assessment based on probability and convex set mixed model, Comput. Struct. 87 (21-22) (2009) 1408-1415.

[31] C. Jiang, et al., Correlation analysis of non-probabilistic convex model and corresponding structural reliability technique, Comput. Methods Appl. Mech. Eng. 200 (33-36) (2011) 2528-2546.

[32] C. Jiang, et al., Structural reliability analysis using non-probabilistic convex model, Comput. Methods Appl. Mech. Eng. 254 (2013) 83-98.

[33] Z. Kang, Y. Luo, A. Li, On non-probabilistic reliability-based design optimization of structures with uncertain-but-bounded parameters, Struct. Saf. 33 (3) (2011) 196-205.

[34] S. Guo, Robust reliability method for non-fragile guaranteed cost control of parametric uncertain systems, Syst. Control Lett. 64 (2014) 27-35.

[35] Z. Meng, et al., A decoupled approach for non-probabilistic reliability-based design optimization, Comput. Struct. 175 (2016) 65-73.

[36] C. Jiang, X. Han, G.R. Liu, Optimization of structures with uncertain constraints based on convex model and satisfaction degree of interval, Comput. Methods Appl. Mech. Eng. 196 (49-52) (2007) 4791-4800.

[37] H. Ishibuchi, H. Tanaka, Multiobjective programming in optimization of the interval objective function, Eur. J. Oper. Res. 48 (2) (1990) 219-225.

[38] X. Wang, Z. Qiu, I. Elishakoff, Non-probabilistic set-theoretic model for structural safety measure, Acta Mech. 198 (1) (2008) 51-64.

[39] F.Y. Li, et al., An uncertain multidisciplinary design optimization method using interval convex models, Eng. Optim. 45 (6) (2013) 697-718.

[40] S.H. Chen, et al., An efficient method for evaluating the natural frequencies of structures with uncertain-but-bounded parameters, Comput. Struct. 87 (9-10) (2009) 582-590.

[41] Z. Qiu, Comparison of static response of structures using convex models and interval analysis method, Int. J. Numer. Methods Eng. 56 (12) (2003) 1735-1753.

[42] J. Sobieszczanski-Sobieski, Sensitivity of complex, internally coupled systems, AIAA J. 28 (1) (1990) 153-160.

[43] X. Du, W. Chen, An efficient approach to probabilistic uncertainty analysis in simulation-based multidisciplinary design, in: 38th Aerospace Sciences Meeting \& Exhibit, 2000.

[44] Z. Qiu, X. Wang, J. Chen, Exact bounds for the static response set of structures with uncertain-but-bounded parameters, Int. J. Solids Struct. 43 (21) (2006) 6574-6593.

[45] J. Tu, K.K. Choi, Y.H. Park, A new study on reliability-based design optimization, J. Mech. Des. 121 (4) (1999) 557-564. 